\title{
THE NEAR-INFRARED VARIABILITY OF A SAMPLE OF OPTICALLY SELECTED QUASARS
}

\author{
G. Neugebauer, B. T. Solfer, K. Matthews, ANd J. H. Elias ${ }^{a}$ \\ Palomar Observatory, California Institute of Technology, Pasadena, California 91125 \\ Received 5 October 1988; revised 18 November 1988
}

\begin{abstract}
A complete sample of 108 optically selected quasars, the Palomar-Green quasars, has been studied at near-infrared wavelengths over an average time base of about $6 \mathrm{yr}$; in some cases the time base extends to $20 \mathrm{yr}$. The measurements show that about half the quasars have a high probability of having varied, but that half show little evidence of variability, in sharp contrast to published studies indicating that most quasars vary on comparable timescales at optical wavelengths. The maximum amplitude of variation is less than $1 \mathrm{mag}$ and generally near $0.5 \mathrm{mag}$. During variations, the near-infrared colors are almost constant. The more luminous quasars show a smaller mean probability of having varied in the time frame of the study than the less-luminous quasars. The majority of the quasars with flat radio spectra have a high probability of having varied. Among those quasars that have a high probability of having varied, the rate is fairly independent of the time interval between the observations after the first year. The quasar 3C 273 shows definite variations at $10.1 \mu \mathrm{m}$ which preclude the emission being thermal emission from heated dust grains for that quasar. The observations of the other quasars cannot be used to differentiate uniquely between thermal and nonthermal emission mechanisms.
\end{abstract}

\section{INTRODUCTION}

Variability has been recognized as a characteristic feature of the radiation observed from quasars since their discovery, and temporal studies of the continuum energy distributions of quasars are potentially important in distinguishing between the physical processes involved in the production of the observed luminosities. In particular, at sufficiently long infrared wavelengths, thermal emission from heated dust can potentially be distinguished from synchrotron radiation by the characteristics of the magnitudes and timescales of variation.

There have been extensive studies of the optical variability of individual quasars, and monitoring of the visible radiation from large samples of selected quasars has been reported by Angione (1971, 1973), Peach (1969), Lu (1972), Selmes, Tritton, and Wordsworth (1975), McGimsey et al. (1975), and Scott et al. (1976). Usher (1978) has investigated the optical variability of quasars in a field of faint blue objects in order to determine the efficacy of identifying quasars through their variability and concludes that essentially all quasars show a high probability of variability on a $20 \mathrm{yr}$ timescale.

The rapid evolution of astronomical infrared observations has meant that published studies of the variability of extragalactic objects at infrared wavelengths have been restricted to relatively short timescales and to studies of few, relatively bright, objects, quite often objects that have exhibited extreme optical variability. Extended monitoring of the nearinfrared infrared flux from selected, generally optically active, quasars has been reported by Neugebauer et al. (1979), Lebofsky and Rieke (1980), Robson et al. (1983, 1986), Bregman et al. (1986), Smith et al. (1987), and Courvoisier et al. $(1987,1988)$.

Because of its brightness, many of the studies relate to the quasar 3C 273. In particular, Rieke and Lebofsky (1979) report that a mild outburst appears to have occurred at 10 $\mu \mathrm{m}$ in early 1972. Robson et al. (1983) have reported the discovery of an infrared flare in 3C 273 in 1983 and Courvoisier et al. (1988) report a short-lived outburst by a factor of 2

\footnotetext{
a) Presently at Cerro Tololo Inter-American Observatory.
}

in the $2.2 \mu \mathrm{m}$ flux of that quasar in early 1988. Cutri et al. (1985) studied the optical and infrared variability of seven "quiescent" quasars over a 3 yr period, and found that over this period of time only one, $3 \mathrm{C} 273$, varied at $2.2 \mu \mathrm{m}$ despite significant variations in the visible and ultraviolet of all the quasars. Further variability of this object at $10 \mu \mathrm{m}$ in 1983 relative to the period 1973-1979 was also reported by Cutri et al.

In this paper we report the results of near-infrared monitoring of a complete sample of 108 optically selected quasars found in the Palomar Green Survey (PG; Green, Schmidt, and Liebert 1986). Although the observations extend over two decades, the main basis of the program is the remeasurement, after a period of about $5 \mathrm{yr}$, of the infrared measurements contained in the study by Neugebauer et al. (1987). The observations were all made using the photometric systems of the Caltech infrared group primarily at the Hale $5 \mathrm{~m}$ Telescope of the Palomar Observatory. The most extensive coverage was obtained at $1.25-2.2 \mu \mathrm{m}$ but observations were also made at 3.5 or $3.7 \mu \mathrm{m}$ and $10.1 \mu \mathrm{m}$.

\section{OBSERVATIONAL DETAILS}

The bulk of the observations were made at the Hale $5 \mathrm{~m}$ Telescope at Palomar Mountain. In addition, a relatively small number of observations, all made before 1980 , were obtained at the Hooker $2.5 \mathrm{~m}$ Telescope on Mount Wilson. Observations at $1.65 \mu \mathrm{m}(H)$ and $2.2 \mathrm{~mm}(K)$ before 1973 were made using a $\mathrm{PbS}$ detector and a focal plane chopper located at the $f / 16$ Cassegrain foci of the telescopes. Observations before 1973 at $3.5 \mu \mathrm{m}(L)$ were obtained with a $\mathrm{PbSe}$ detector. Observations made after 1973 at $1.25 \mu \mathrm{m}(J), 1.65$ $\mu \mathrm{m}, 2.2 \mu \mathrm{m}, 3.5 \mu \mathrm{m}$, and $3.7 \mu \mathrm{m}\left(L^{\prime}\right)$ were made with an InSb detector; the change to $3.7 \mu \mathrm{m}$ was made in 1980 . Before 1970 , measurements were often made at only $2.2 \mu \mathrm{m}$; after 1973, measurements at 1.25 and $1.65 \mu \mathrm{m}$ generally accompanied measurements at $2.2 \mu \mathrm{m}$. Observations at 10.1 $\mu \mathrm{m}(N)$ were made with Ge:Ga bolometers. Whenever colors are given below, observations at the different wavelengths were made on the same night.

Observations before 1977 were made with a photometer using a focal plane chopper. The beam diameters were typi- 
cally $5^{\prime \prime}-10^{\prime \prime}$, but extended up to $15^{\prime \prime}$. The spacing between the two beams used to cancel sky emission, the "chopper throw," was typically $15^{\prime \prime}$ at the Hale Telescope and 30 " on the Hooker Telescope. After 1977, observations at the Hale $5 \mathrm{~m}$ Telescope were made using the $f / 70$ Gregorian or Cassegrain focus and a "chopping" secondary mirror with throws which typically were $15^{\prime \prime}$. Beam diameters are limited to be less than $10^{\prime \prime}$ at wavelengths less than $3.7 \mu \mathrm{m}$ and $5^{\prime \prime}$ at 10.1 $\mu \mathrm{m}$. Since 1985 , observations from 1.25 to $2.2 \mu \mathrm{m}$ have been obtained with $5 "$ and $10^{\prime \prime}$ diameter beams on each night of observation whenever possible.

The photometry for wavelengths up to $3.7 \mu \mathrm{m}$ was calibrated using a set of secondary standard stars which evolved into the set described by Elias et al. (1982). The calibration stars used for the $10 \mu \mathrm{m}$ photometry are consistent with those described by Tokunaga (1984). Typically, between four and ten calibration stars were observed on each night that quasars in the present sample were measured. As an example of the stability of the Caltech photometry, $107 \mathrm{ob}-$ servations of the $K=8.8$ mag star Bertaud b near BL Lac (Bertaud et al. 1973) obtained from November 1969 through July 1988 show a change in the biannual averages of less than $5 \%$ with a dispersion in the sample of $4 \%$. This star has been monitored in a longstanding program of observations of BL Lac and observations of it were obtained under the same conditions as applied to the PG quasars.

No correction for differing photometry because of the different detectors or filters was made except that the $3.5 \mu \mathrm{m}$ magnitudes were made brighter by 0.15 mag to place them on the $L^{\prime}$ system, and measurements at both wavelengths will be lumped together as " $3.7 \mu \mathrm{m}$ " observations. The effects at the other wavelengths are estimated to be significantly less than $0.05 \mathrm{mag}$.

The distributions of the uncertainties over the total span of measurements at $2.2 \mu \mathrm{m}$, which is typical of that at 1.25 and $1.65 \mu \mathrm{m}$, at $3.7 \mu \mathrm{m}$ and at $10.1 \mu \mathrm{m}$, are given in Fig. 1 . Most of the PG quasars are bright enough at 1.25-2.2 $\mu \mathrm{m}$ that the statistical uncertainties at these wavelengths were generally on the order of $1 \%-2 \%$ and the uncertainties in the photometry are due to systematic effects such as beam profiles, centering, extinction corrections, and overall calibration with respect to the secondary standards. These systematic effects, which since about 1977 are on the order of $5 \%$, were larger before that time when the techniques for infrared photometry were being developed. We have, based on the observations of Bertaud b (Bertaud et al. 1973) and of the secondary calibration stars, included a systematic uncertainty of $7 \%$ in quadrature with the statistical uncertainties at $1.25,1.65$, and $2.2 \mu \mathrm{m}$ for observations made prior to 1973. The earlier measurements with larger uncertainties were kept in the database in order to preserve, although with reduced accuracy, the longest-time histories.

The uncertainties at $3.7 \mu \mathrm{m}$ are generally dominated by the counting statistics and usually exceed $6 \%$. These uncertainties also dominate any additional change in the photometry that resulted from the change in effective wavelength from 3.5 to $3.7 \mu \mathrm{m}$. At $10.1 \mu \mathrm{m}$, the uncertainties were almost always dominated by the statistical uncertainties, which generally exceeded $10 \%$. In the analysis, a limiting maximum uncertainty, appropriate to the particular analysis, was often invoked.

As stated above, the observations were made with beam sizes ranging from 5 " to $15^{\prime \prime}$ in diameter depending on the atmospheric seeing, the equipment in use, and the observa-

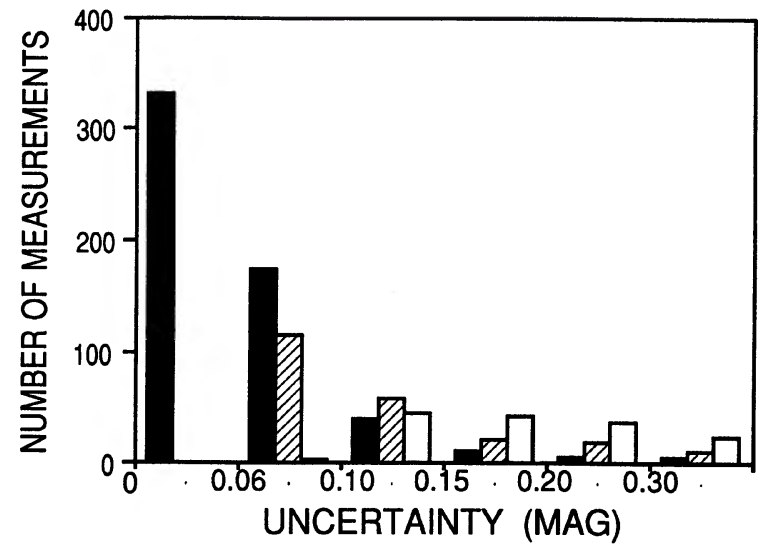

FIG. 1. Histograms of the total measurement uncertainties in the observations presented here are shown. The uncertainties for observations at $2.2 \mu \mathrm{m}$ are at the left of each group (dark), and are typical for observations at 1.25 and $1.65 \mu \mathrm{m}$. The middle bars (hatched) indicate the uncertainties at $3.7 \mu \mathrm{m}$, while the right-hand bars (light) give the distributions of the uncertainties at $10.1 \mu \mathrm{m}$. The uncertainties are given in magnitudes.

tional program in progress. A comparison of measurements made with different beam sizes can introduce artificial variability if a significant flux from a host galaxy is included in the measurements obtained with the larger beams. Alternatively, if only large beam measurements were obtained, the host galaxy, which is presumably constant in time, could have the effect of hiding variability in the underlying quasar. This effect is presumed to be significant relative to the uncertainties only at wavelengths from 1.25 to $2.2 \mu \mathrm{m}$. The vast majority of the measurements at these wavelengths were made with beam diameters between $5^{\prime \prime}$ and 10"; e.g., the observations in 1980-1981, which form the basis of Neugebauer et al. (1987), were all done with 5.5" diameter beams and subsequent observations were made with either 5 " or $10^{\prime \prime}$ diameter beams or both.

In order to determine the magnitude of artifacts due to the presence of an accompanying galaxy, detailed measurements of 16 PG quasars were made through annular diaphragms of inner diameter 5" and outer diameter 10" (Neugebauer et al. 1985) and observations since $\sim 1985$ at $1.25-$ $2.2 \mu \mathrm{m}$ have been made using both $5^{\prime \prime}$ and $10^{\prime \prime}$ diameter beams whenever permitted by the seeing. These measurements, which are shown in Fig. 2, indicate that the host galaxies can contribute as much as $40 \%$ of the total measured in a 5" diameter beam, although the typical value is less than $10 \%$. Furthermore, it is seen in Fig. 2 that significant extended emission is measured only for quasars with redshifts $z$ less than 0.35 .

Seventy-one quasars in the sample considered here have redshifts less than 0.35 . Of these, 67 , including all sample quasars with $z<0.1$, have been re-observed with $5^{\prime \prime}$ and 10" diameter beams at $1.25,1.65$, and $2.2 \mu \mathrm{m}$ on at least one night or were measured using the 5" and 10" annular diaphragm. For these 67 quasars, the $1.25-2.2 \mu \mathrm{m}$ measurements made with beams larger than $5^{\prime \prime}$ in diameter were revised to take account of the host galaxy with the assumption that the galaxy contribution was as determined from the multibeam measurements. When measurements with 5 " and 

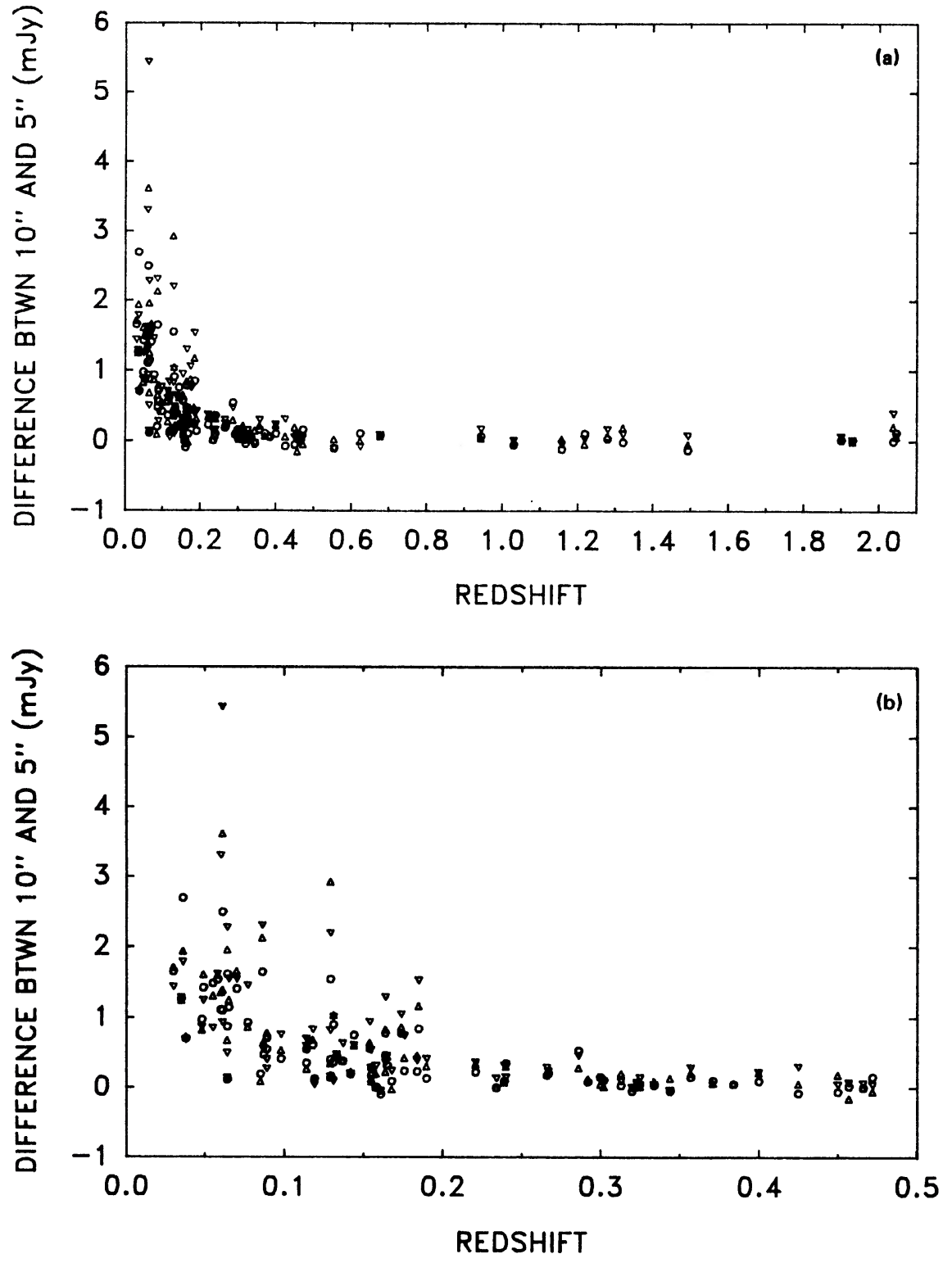

FiG. 2. (a) The strength of the galaxy surrounding the quasar is indicated by the difference in flux density as measured with a beam $10^{\prime \prime}$ in diameter and one 5 " in diameter. Measurements at $1.25 \mu \mathrm{m}$ are shown by light circles, measurements at $1.65 \mu \mathrm{m}$ as triangles, and measurements at $2.2 \mu \mathrm{m}$ as inverted triangles. All the measurements of this difference obtained during this study are included. (b) Same as (a), but redshifts limited to those less than 0.5 .
10 " diameter beams were made on one night, the measurements made with the smaller beam were used in the analysis. For the remaining four quasars with redshifts less than 0.35 , the measurements with large beams were corrected assuming a flux density versus redshift relation corresponding to a smooth median of the flux densities observed at all three wavelengths and displayed in Fig. 2. It was assumed that no correction was required for quasars with redshifts larger than 0.35 . The increased uncertainties associated with these corrections are included in Fig. 1. No correction to the flux density inside the $5^{\prime \prime}$ diameter beam was attempted since, within the uncertainties of the measurements, simple models of galaxies centered on the quasars proved inconsistent with the observations.

\section{THE SAMPLE}

The present sample consists of 108 quasars selected from the Palomar Green Survey of objects with an ultraviolet ex- cess (Green, Schmidt, and Liebert 1986). Thus, since the PG survey represents an unbiased sample of optically selected quasars, the current sample should characterize the infrared variability of such a sample. One hundred five of the 108 quasars were observed in 1980-1981 at visual and nearinfrared wavelengths in the study by Neugebauer et al. (1987). All the quasars included in Neugebauer et al. were subsequently remeasured in the 1985-1988 time frame. In addition, three quasars (PG $0050+124=1 Z$ W 1, PG 1322 +659 , and PG $1425+267=$ TON 202), which were arbitrarily omitted from Neugebauer et al., but which were selected as part of the Palomar Green survey, have been included in the present sample. The quasars belonging to the present sample are listed in Table I.

Of the 108 subjects in Table $\mathrm{I}$, all but seven are included in the bright quasar survey of Schmidt and Green (1983). The seven additional quasars in Table I, which were included in Neugebauer et al. (1987), were also found in the Palomar Green survey, but lie outside the area limits for completeness 
set by Schmidt and Green. The objects in the present sample for variability thus share with the objects in the bright quasar sample the morphological criterion that the objects should have a dominant starlike appearance on the blue prints of the first Palomar 48 in. Schmidt Sky Survey and the spectroscopic criterion that the object show broad emission lines with substantial redshift.

No strict luminosity criterion was applied in the selection of objects by Schmidt and Green (1983), but these authors differentiate between objects brighter than $M_{B}=-23$ mag $\left(H_{0}=50 \mathrm{~km} \mathrm{~s}^{-1} \mathrm{Mpc}^{-1}\right)$, the "quasars," and objects fainter than $M_{B}=23 \mathrm{mag}$, the "low-luminosity quasars." Of the 108 quasars in Table I, 94 are, by this definition, quasars and 14 are "low-luminosity quasars." If, as assumed hereafter, $H_{0}$ is taken as $75 \mathrm{~km} \mathrm{~s}^{-1} \mathrm{Mpc}^{-1}$, the demarcation of Schmidt and Green corresponds to $M_{B}=-22.1 \mathrm{mag}$ or

TABLE I. Log of observations

\begin{tabular}{|c|c|c|c|c|c|c|c|c|c|c|c|c|}
\hline \multirow[t]{2}{*}{ Object } & \multirow[t]{2}{*}{$\begin{array}{l}\text { Radio } \\
\text { Type }^{1}\end{array}$} & \multicolumn{5}{|c|}{$\begin{array}{l}\text { Number of Measurements } \\
\text { at Wavelength }\end{array}$} & \multicolumn{3}{|c|}{ Variability at $2.2 \mu \mathrm{m}^{2}$} & \multicolumn{3}{|c|}{ Variability at $10.1 \mu \mathrm{m}^{2}$} \\
\hline & & 1.25 & 1.65 & $\begin{array}{c}2.2 \\
(\mu \mathrm{m})\end{array}$ & 3.7 & $10.1^{3}$ & $\begin{array}{l}\text { span } \\
\text { (yrs) }\end{array}$ & $\begin{array}{l}\text { disp } \\
\text { (mag) }\end{array}$ & $P_{v}(2.2)$ & $\begin{array}{l}\text { span } \\
\text { (yrs) }\end{array}$ & $\begin{array}{l}\text { disp } \\
\text { (mag) }\end{array}$ & $P_{v}(10.1)$ \\
\hline $0002+051$ & $\mathbf{L}$ & 5 & 5 & 5 & 1 & 0 & 6.2 & 0.09 & 0.825 & & & \\
\hline $0003+158$ & L & 3 & 3 & 5 & 0 & 0 & 19.2 & 0.10 & 0.838 & & & \\
\hline $0007+106$ & $\mathbf{F}$ & 26 & 28 & 29 & 13 & 9 & 18.0 & 0.23 & 1.000 & 10.9 & 0.14 & 0.365 \\
\hline $0026+129$ & $\mathbf{Q}$ & 6 & 7 & 7 & 4 & 3 & 11.0 & 0.10 & 0.997 & 10.1 & 0.14 & 0.296 \\
\hline $0043+039$ & $\mathbf{Q}$ & 3 & 3 & 3 & 0 & 0 & 6.0 & 0.03 & 0.284 & & & \\
\hline $0044+030$ & $\mathbf{F}$ & 2 & 2 & 2 & 0 & 0 & 6.0 & 0.03 & 0.552 & & & \\
\hline $0049+171$ & $\mathbf{Q}$ & 3 & 3 & 3 & 1 & 0 & 7.2 & 0.38 & 1.000 & & & \\
\hline $0050+124$ & $\mathbf{Q}$ & 1 & 5 & 5 & 3 & 3 & 18.8 & 0.10 & 0.947 & 11.8 & 0.09 & 0.383 \\
\hline $0052+251$ & $\mathbf{Q}$ & 4 & 4 & 4 & 3 & 2 & 6.0 & 0.05 & 0.640 & 4.3 & 0.16 & 0.648 \\
\hline $0117+213$ & $\mathbf{Q}$ & 3 & 3 & 3 & 2 & 2 & 5.8 & 0.05 & 0.574 & 2.8 & 0.04 & 0.140 \\
\hline $0157+001$ & A & 4 & 4 & 4 & 3 & 3 & 5.9 & 0.04 & 0.527 & 4.9 & 0.09 & 0.479 \\
\hline $0804+761$ & $\mathbf{Q}$ & 6 & 6 & 6 & 3 & 2 & 5.7 & 0.09 & 0.997 & 4.0 & 0.05 & 0.339 \\
\hline $0832+251$ & A & 5 & 5 & 5 & 3 & 1 & 7.1 & 0.04 & 0.294 & & & \\
\hline $0838+770$ & $\mathbf{Q}$ & 7 & 7 & 7 & 3 & 1 & 5.3 & 0.09 & 0.994 & & & \\
\hline $0844+349$ & $\mathbf{Q}$ & 5 & 5 & 6 & 5 & 2 & 6.4 & 0.05 & 0.445 & 4.3 & 0.07 & 0.503 \\
\hline $0906+484$ & A & 8 & 8 & 8 & 3 & 2 & 10.4 & 0.12 & 1.000 & 10.4 & 0.01 & 0.053 \\
\hline $0923+201$ & $\mathbf{Q}$ & 4 & 4 & 4 & 3 & 1 & 7.5 & 0.10 & 0.995 & & & \\
\hline $0931+437$ & $\mathbf{A}$ & 6 & 6 & 6 & 3 & 1 & 7.8 & 0.07 & 0.654 & & & \\
\hline $0935+417$ & A & 4 & 4 & 4 & 1 & 0 & 7.8 & 0.03 & 0.152 & & & \\
\hline $0946+301$ & $\mathbf{Q}$ & 2 & 2 & 2 & 0 & 0 & 5.3 & 0.04 & 0.581 & & & \\
\hline $0947+396$ & $\mathbf{Q}$ & 2 & 2 & 2 & 0 & 0 & 5.7 & 0.10 & 0.924 & & & \\
\hline $0953+414$ & $\mathbf{Q}$ & 6 & 6 & 6 & 2 & 1 & 9.9 & 0.14 & 1.000 & & & \\
\hline $1001+054$ & $\mathbf{Q}$ & 4 & 4 & 4 & 1 & 1 & 11.4 & 0.31 & 1.000 & & & \\
\hline $1004+130$ & $\mathbf{F}$ & 5 & 5 & 5 & 4 & 2 & 10.2 & 0.07 & 0.847 & 10.2 & 0.17 & 0.621 \\
\hline $1008+133$ & Q & 4 & 4 & 4 & 2 & 2 & 7.1 & 0.02 & 0.056 & 0.4 & 0.00 & 0.005 \\
\hline $1011-040$ & $\mathbf{Q}$ & 3 & 3 & 3 & 1 & 1 & 7.1 & 0.07 & 0.950 & & & \\
\hline $1012+008$ & $\mathbf{Q}$ & 3 & 3 & 3 & 1 & 1 & 7.1 & 0.08 & 0.974 & & & \\
\hline $1048-090$ & $\mathbf{F}$ & 2 & 2 & 2 & 0 & 0 & 6.1 & 0.09 & 0.966 & & & \\
\hline $1048+342$ & $\mathbf{Q}$ & 2 & 2 & 2 & 0 & 0 & 5.3 & 0.06 & 0.801 & & & \\
\hline 1049-005 & $\mathbf{Q}$ & 3 & 3 & 3 & 1 & 1 & 7.1 & 0.06 & 0.733 & & & \\
\hline $1100+772$ & L & 4 & 4 & 5 & 2 & 0 & 19.9 & 0.05 & 0.389 & & & \\
\hline $1103-006$ & $\mathbf{F}$ & 2 & 2 & 2 & 0 & 0 & 6.1 & 0.02 & 0.412 & & & \\
\hline $1112+431$ & A & 5 & 5 & 5 & 3 & 1 & 7.9 & 0.32 & 1.000 & & & \\
\hline $1114+445$ & $\mathbf{Q}$ & 4 & 4 & 4 & 3 & 1 & 7.9 & 0.07 & 0.728 & & & \\
\hline $1115+080$ & $\mathbf{Q}$ & 2 & 2 & 2 & 0 & 0 & 4.9 & 0.04 & 0.671 & & & \\
\hline $1115+407$ & Q & 3 & 3 & 3 & 0 & 0 & 8.1 & 0.01 & 0.021 & & & \\
\hline $1116+215$ & $\mathbf{Q}$ & 3 & 3 & 3 & 2 & 1 & 7.9 & 0.05 & 0.696 & & & \\
\hline $1119+120$ & $\mathbf{Q}$ & 5 & 5 & 5 & 4 & 3 & 8.0 & 0.08 & 0.935 & 7.1 & 0.02 & 0.037 \\
\hline $1121+422$ & $\mathbf{Q}$ & 3 & 3 & 3 & 0 & 0 & 8.1 & 0.05 & 0.413 & & & \\
\hline $1126-041$ & $\mathbf{Q}$ & 3 & 3 & 3 & 2 & 1 & 6.7 & 0.04 & 0.631 & & & \\
\hline $1138+040$ & A & 2 & 2 & 2 & 0 & 0 & 7.1 & 0.01 & 0.222 & & & \\
\hline $1148+549$ & $\mathbf{Q}$ & 3 & 3 & 3 & 1 & 1 & 7.1 & 0.04 & 0.446 & & & \\
\hline $1151+117$ & $\overline{\mathbf{A}}$ & 2 & 2 & 2 & 0 & 0 & 6.9 & 0.04 & 0.695 & & & \\
\hline
\end{tabular}


TABLE I. (continued)

\begin{tabular}{|c|c|c|c|c|c|c|c|c|c|c|c|c|}
\hline \multirow[t]{2}{*}{ Object } & \multirow[t]{2}{*}{$\begin{array}{l}\text { Radio } \\
\text { Type }^{1}\end{array}$} & \multicolumn{5}{|c|}{$\begin{array}{l}\text { Number of Measurements } \\
\text { at Wavelength }\end{array}$} & \multicolumn{3}{|c|}{ Variability at $2.2 \mu \mathrm{m}^{2}$} & \multicolumn{3}{|c|}{ Variability at $10.1 \mu \mathrm{m}^{2}$} \\
\hline & & 1.25 & 1.65 & $\begin{array}{c}2.2 \\
(\mu \mathrm{m})\end{array}$ & 3.7 & $10.1^{3}$ & $\begin{array}{l}\text { span } \\
\text { (yrs) }\end{array}$ & $\begin{array}{l}\text { disp } \\
\text { (mag) }\end{array}$ & $P_{v}(2.2)$ & $\begin{array}{c}\text { span } \\
\text { (yrs) }\end{array}$ & $\begin{array}{l}\text { disp } \\
\text { (mag) }\end{array}$ & $P_{v}(10.1)$ \\
\hline $1202+281$ & $\mathbf{Q}$ & 3 & 6 & 6 & 2 & 1 & 6.8 & 0.13 & 1.000 & & & \\
\hline $1206+459$ & $\mathbf{Q}$ & 3 & 3 & 3 & 1 & 1 & 6.7 & 0.03 & 0.248 & & & \\
\hline $1211+143$ & & 6 & 6 & 6 & 3 & 2 & 8.1 & 0.07 & 0.953 & 5.8 & 0.00 & 0.000 \\
\hline $1216+069$ & $\mathbf{Q}$ & 2 & 2 & 2 & 0 & 0 & 6.1 & 0.03 & 0.604 & & & \\
\hline $1222+228$ & $\mathbf{A}$ & 5 & 5 & 5 & 0 & 0 & 6.9 & 0.03 & 0.027 & & & \\
\hline $1226+023$ & $\mathbf{F}$ & 51 & 87 & 95 & 41 & 26 & 21.1 & 0.09 & 1.000 & 14.2 & 0.16 & 0.789 \\
\hline $1229+204$ & $\mathbf{Q}$ & 4 & 4 & 5 & 3 & 2 & 19.0 & 0.09 & 0.960 & 5.8 & 0.13 & 0.844 \\
\hline $1241+176$ & L & 2 & 2 & 2 & 0 & 0 & 6.8 & 0.05 & 0.620 & & & \\
\hline $1244+026$ & $\mathbf{Q}$ & 2 & 2 & 2 & 0 & 0 & 6.8 & 0.12 & 1.000 & & & \\
\hline $1247+267$ & $\mathbf{Q}$ & 2 & 2 & 2 & 0 & 0 & 6.9 & 0.01 & 0.222 & & & \\
\hline $1248+401$ & $\mathbf{Q}$ & 3 & 3 & 3 & 1 & 1 & 7.1 & 0.05 & 0.573 & & & \\
\hline $1254+047$ & $\mathbf{Q}$ & 2 & 2 & 2 & 0 & 0 & 6.1 & 0.05 & 0.722 & & & \\
\hline $1259+593$ & $\mathbf{Q}$ & 2 & 2 & 2 & 0 & 0 & 6.9 & 0.05 & 0.610 & & & \\
\hline $1302-102$ & $\mathbf{F}$ & 2 & 2 & 2 & 0 & 0 & 6.1 & 0.11 & 0.997 & & & \\
\hline $1307+085$ & $\mathbf{Q}$ & 6 & 6 & 6 & 2 & 1 & 8.1 & 0.08 & 0.929 & & & \\
\hline $1309+355$ & L & 2 & 2 & 2 & 0 & 0 & 7.0 & 0.12 & 1.000 & & & \\
\hline $1310-108$ & $\mathbf{Q}$ & 2 & 2 & 2 & 0 & 0 & 6.9 & 0.05 & 0.843 & & & \\
\hline $1322+659$ & $\mathbf{Q}$ & 2 & 2 & 2 & 0 & 0 & 6.9 & 0.08 & 0.960 & & & \\
\hline $1329+412$ & $\mathbf{Q}$ & 2 & 2 & 2 & 0 & 0 & 6.9 & 0.14 & 0.998 & & & \\
\hline $1333+176$ & L & 2 & 2 & 2 & 0 & 0 & 6.9 & 0.01 & 0.193 & & & \\
\hline $1338+416$ & $\mathbf{Q}$ & 2 & 2 & 2 & 0 & 0 & 6.9 & 0.05 & 0.729 & & & \\
\hline $1351+236$ & $\mathbf{Q}$ & 2 & 2 & 2 & 0 & 0 & 6.9 & 0.09 & 0.989 & & & \\
\hline $1351+640$ & A & 8 & 9 & 9 & 4 & 5 & 13.3 & 0.04 & 0.147 & 12.1 & 0.11 & 0.401 \\
\hline $1352+011$ & $\mathbf{Q}$ & 3 & 3 & 3 & 0 & 0 & 6.9 & 0.05 & 0.466 & & & \\
\hline $1352+183$ & $\mathbf{Q}$ & 2 & 2 & 2 & 0 & 0 & 6.9 & 0.19 & 1.000 & & & \\
\hline $1354+213$ & A & 2 & 2 & 2 & 0 & 0 & 6.1 & 0.10 & 0.990 & & & \\
\hline $1402+261$ & $\mathbf{Q}$ & 3 & 3 & 3 & 1 & 1 & 7.9 & 0.05 & 0.697 & & & \\
\hline $1404+226$ & $\mathbf{Q}$ & 2 & 2 & 2 & 0 & 0 & 6.9 & 0.14 & 1.000 & & & \\
\hline $1407+265$ & A & 4 & 4 & 4 & 0 & 0 & 6.9 & 0.10 & 0.853 & & & \\
\hline $1411+442$ & $\mathbf{Q}$ & 3 & 3 & 3 & 2 & 2 & 6.2 & 0.12 & 0.999 & 5.2 & 0.12 & 0.701 \\
\hline $1415+451$ & $\mathbf{Q}$ & 3 & 3 & 3 & 0 & 0 & 8.1 & 0.02 & 0.221 & & & \\
\hline $1416-129$ & $\mathbf{Q}$ & 2 & 2 & 2 & 0 & 0 & 6.9 & 0.02 & 0.304 & & & \\
\hline $1425+267$ & $\mathbf{F}$ & 1 & 4 & 6 & 3 & 0 & 18.1 & 0.19 & 0.997 & & & \\
\hline $1426+015$ & $\mathbf{Q}$ & 3 & 3 & 3 & 2 & 2 & 6.1 & 0.11 & 0.997 & 5.0 & 0.19 & 0.910 \\
\hline $1427+480$ & $\mathbf{Q}$ & 3 & 3 & 3 & 0 & 0 & 8.1 & 0.04 & 0.580 & & & \\
\hline $1435-067$ & $\mathbf{Q}$ & 3 & 3 & 3 & 0 & 0 & 6.9 & 0.03 & 0.462 & & & \\
\hline $1440+356$ & $\mathbf{Q}$ & 5 & 5 & 5 & 3 & 2 & 6.2 & 0.05 & 0.677 & 5.2 & 0.08 & 0.532 \\
\hline $1444+407$ & $\mathbf{Q}$ & 4 & 4 & 4 & 1 & 1 & 8.1 & 0.03 & 0.231 & & & \\
\hline $1448+273$ & $\mathbf{Q}$ & 4 & 4 & 4 & 2 & 2 & 6.2 & 0.04 & 0.443 & 5.2 & 0.12 & 0.570 \\
\hline $1501+106$ & Q & 6 & 6 & 6 & 3 & 2 & 6.2 & 0.12 & 1.000 & 5.0 & 0.00 & 0.000 \\
\hline $1512+370$ & $\mathbf{L}$ & 3 & 3 & 4 & 2 & 1 & 10.3 & 0.11 & 0.940 & & & \\
\hline $1519+226$ & $\mathbf{Q}$ & 3 & 3 & 4 & 1 & 0 & 8.8 & 0.03 & 0.205 & & & \\
\hline $1522+101$ & $\mathbf{Q}$ & 3 & 3 & 3 & 2 & 2 & 5.4 & 0.05 & 0.409 & 4.1 & 0.08 & 0.294 \\
\hline $1534+580$ & $\mathbf{Q}$ & 4 & 4 & 4 & 2 & 1 & 8.1 & 0.10 & 0.996 & & & \\
\hline $1535+547$ & $\mathbf{Q}$ & 8 & 10 & 10 & 4 & 4 & 17.2 & 0.12 & 1.000 & 12.2 & 0.12 & 0.384 \\
\hline $1543+489$ & $\mathbf{Q}$ & 2 & 2 & 2 & 1 & 1 & 7.3 & 0.02 & 0.293 & & & \\
\hline $1545+210$ & L & 5 & 5 & 6 & 0 & 0 & 19.8 & 0.09 & 0.992 & & & \\
\hline $1552+085$ & $\mathbf{Q}$ & 4 & 4 & 4 & 2 & 1 & 8.1 & 0.06 & 0.791 & & & \\
\hline
\end{tabular}


TABLE I. (continued)

\begin{tabular}{|c|c|c|c|c|c|c|c|c|c|c|c|c|}
\hline \multirow[t]{2}{*}{ Object } & \multirow[t]{2}{*}{$\begin{array}{l}\text { Radio } \\
\text { Type }^{1}\end{array}$} & \multicolumn{5}{|c|}{$\begin{array}{l}\text { Number of Measurements } \\
\text { at Wavelength }\end{array}$} & \multicolumn{3}{|c|}{ Variability at $2.2 \mu \mathrm{m}^{2}$} & \multicolumn{3}{|c|}{ Variability at $10.1 \mu \mathrm{m}^{2}$} \\
\hline & & 1.25 & 1.65 & $\begin{array}{c}2.2 \\
(\mu \mathrm{m})\end{array}$ & & $10.1^{3}$ & $\begin{array}{l}\text { span } \\
\text { (yrs) }\end{array}$ & $\begin{array}{l}\text { disp } \\
\text { (mag) }\end{array}$ & $P_{v}(2.2)$ & $\begin{array}{l}\text { span } \\
\text { (yrs) }\end{array}$ & $\begin{array}{l}\text { disp } \\
\text { (mag) }\end{array}$ & $P_{v}(10.1)$ \\
\hline $1612+261$ & $\mathbf{A}$ & 5 & 5 & 8 & 2 & 2 & 19.2 & 0.13 & 0.997 & 10.3 & 0.04 & 0.146 \\
\hline $1613+658$ & $\mathbf{Q}$ & 4 & 4 & 4 & 3 & 2 & 6.4 & 0.13 & 1.000 & 5.2 & 0.22 & 0.840 \\
\hline $1617+175$ & $\mathbf{Q}$ & 5 & 5 & 5 & 2 & 2 & 8.1 & 0.11 & 1.000 & 4.7 & 0.06 & 0.323 \\
\hline $1626+554$ & $\mathbf{Q}$ & 3 & 3 & 3 & 1 & 1 & 8.1 & 0.07 & 0.906 & & & \\
\hline $1630+377$ & $\mathbf{Q}$ & 6 & 6 & 6 & 1 & 0 & 6.4 & 0.06 & 0.366 & & & \\
\hline $1634+706$ & $\mathbf{Q}$ & 5 & 5 & 5 & 2 & 2 & 6.0 & 0.03 & 0.107 & 4.8 & 0.11 & 0.525 \\
\hline $1700+518$ & $\mathbf{Q}$ & 7 & 7 & 7 & 4 & 4 & 7.9 & 0.03 & 0.052 & 7.0 & 0.06 & 0.118 \\
\hline $1704+608$ & L & 8 & 8 & 12 & 4 & 5 & 21.0 & 0.16 & 1.000 & 11.6 & 0.19 & 0.652 \\
\hline $1718+481$ & $\mathbf{L}$ & 5 & 5 & 5 & 2 & 2 & 4.7 & 0.02 & 0.015 & 4.3 & 0.04 & 0.157 \\
\hline $2112+059$ & $\mathbf{Q}$ & 4 & 4 & 4 & 3 & 2 & 6.1 & 0.04 & 0.234 & 5.0 & 0.11 & 0.446 \\
\hline $2130+099$ & $\mathbf{Q}$ & 4 & 6 & 6 & 4 & 3 & 16.9 & 0.13 & 1.000 & 9.8 & 0.10 & 0.514 \\
\hline $2209+184$ & $\mathbf{L}$ & 5 & 5 & 6 & 2 & 3 & 6.1 & 0.12 & 1.000 & 4.9 & 0.05 & 0.020 \\
\hline $2233+134$ & $\mathbf{Q}$ & 7 & 7 & 7 & 5 & 1 & 7.3 & 0.04 & 0.435 & & & \\
\hline $2251+113$ & $\mathbf{L}$ & 4 & 4 & 7 & 3 & 0 & 19.1 & 0.06 & 0.364 & & & \\
\hline $2302+029$ & $\mathbf{Q}$ & 11 & 11 & 11 & 5 & 4 & 6.0 & 0.07 & 0.621 & 4.4 & 0.32 & 0.834 \\
\hline $2344+092$ & $\mathbf{F}$ & 5 & 5 & 8 & 4 & 1 & 20.0 & 0.12 & 0.920 & & & \\
\hline $2349-014$ & $\mathbf{F}$ & 5 & 5 & 5 & 3 & 1 & 7.2 & 0.12 & 1.000 & & & \\
\hline
\end{tabular}

${ }^{1} \mathrm{~L}-\log \left[\mathrm{f}_{\nu}(6 \mathrm{~cm}) / \mathrm{f}_{\nu}(1.25 \mu \mathrm{m})\right]>4.5$

$F-\log \left[f_{\nu}(6 \mathrm{~cm}) / f_{\nu}(1.25 \mu \mathrm{m})\right]>4.5, \alpha_{\mathrm{r}}>-.04$

$Q-\log \left[f_{\nu}(6 \mathrm{~cm}) / \mathrm{f}_{\nu}(1.25 \mu \mathrm{m})\right]<3.5$

$A-3.5<\log \left[f_{\nu}(6 \mathrm{~cm}) / \mathrm{f}_{\nu}(1.25 \mu \mathrm{m})\right]<4.5$

${ }^{2}$ span $=$ maximum time separation

disp $=$ dispersion from mean

$P_{v}=$ probability of having varied

${ }^{3}$ Limits and observations with $>0.5$ mag uncertainties are not included.

a blue luminosity $\log \left(L(0.44)\left[L_{\odot}\right]\right)=10.8$. The blue luminosity is taken as $L(0.44)=v f_{v}(0.44)$ where $v$ is the frequency of blue light and $f_{v}(0.44)$ is the flux density at 0.44 $\mu \mathrm{m}$; luminosities at other wavelengths are defined similarly.

Sanders et al. (1988), using IRAS data from 12 to $100 \mu \mathrm{m}$ plus new $10.1 \mu \mathrm{m}$ observations, have determined the integrated luminosity from 0.3 to $300 \mu \mathrm{m}$ for 64 quasars in the Palomar Green sample and thus can estimate a bolometric luminosity for those quasars and the ratio of the bolometric luminosity to that at 0.44 and $2.2 \mu \mathrm{m}$. On the basis of these estimates, the demarcation assumed by Schmidt and Green (1983) corresponds to a bolometric luminosity $\log (L(\mathrm{bol})$ $\left.\left[L_{\odot}\right]\right)=12.0$, while the ratio of the bolometric luminosity to that at $2.2 \mu \mathrm{m}$, which is available for all the objects, is $L($ bol) $/ L(2.2)=18$ with a standard deviation (population ) of $30 \%$. This ratio will be used to estimate the bolometric luminosity of those quasars for which IRAS detections are not available. The number distributions of the quasars in the sample as a function of $L(2.2)$ and $L(\mathrm{bol})$ are shown in Fig. 3. The mean $2.2 \mu \mathrm{m}$ magnitudes observed in the present study were used to obtain $L(2.2)$, while $L(\mathrm{bol})$ was either obtained from Sanders et al. or calculated from $L(2.2)$.
RESULTS-OBSERVATIONAL

Most of the observations that give variability information were made at wavelengths from 1.25 to $2.2 \mu \mathrm{m}$, and the fewest were made at $10.1 \mu \mathrm{m}$. Multiple observations at 1.25 , 1.65 , and $2.2 \mu \mathrm{m}$ were obtained for all 108 quasars, while only 54 and 29 of the sample had significant multiple observations at 3.7 and $10.1 \mu \mathrm{m}$, respectively. The distribution of the number of measurements at $2.2 \mu \mathrm{m}$ for each quasar is displayed in Fig. 4 and the number of measurements at all wavelengths is included in Table I.*

As indicated in Fig. 4, most of the quasars were measured more often than in the two periods 1980-1981 and 19851988. In some cases, e.g., PG $1226+023=3 \mathrm{C} 273$, the qua-

*See AIP document no. PAPS ANJOA-97-957-25 for 20 pages of Table of Individual Observations at $1.25,1.65,2.2,3.7$, and $10.1 \mu \mathrm{m}$ for 108 Quasars. Order by PAPS number and journal reference from American Institute of Physics, Physics Auxiliary Publication Service, 335 East 45th Street, New York, NY 10017 . The price is $\$ 1.50$ for each microfiche (98 pages) or $\$ 5.00$ for photocopies of up to 30 pages, and $\$ 0.15$ for each additional page over 30 pages. Airmail additional. Make checks payable to the American Institute of Physics. 


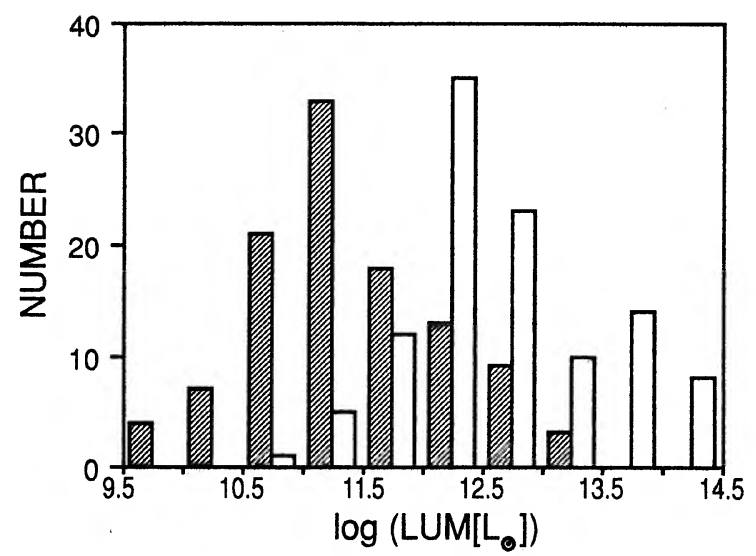

FIG. 3. The distributions of the $2.2 \mu \mathrm{m}$ and bolometric luminosities, $L(2.2)$ and $L(\mathrm{bol})$, are presented for 70 of the PG quasars. The distribution of $L(2.2)$ is shown by the left-hand bars in each group (hatched), while that of $L(\mathrm{bol})$ is indicated by the right-hand bars (light). The bolometric luminosities are from Sanders et al. (1988) or are derived from $L(2.2)$ as described in the text.

sars were known to be bright or otherwise thought to be interesting and were measured prior to 1980, some as early as 1968. None of the PG quasars, with the possible exception of PG $0007+106=3 Z W 2$, were selected for monitoring primarily because they were known to be highly variable. Some quasars were remeasured after 1981 to obtain observations at 3.7 and $10.1 \mu \mathrm{m}$, and in each case the $1.25-2.2 \mu \mathrm{m}$ measurements were repeated. Still other PG quasars were remeasured in order to determine the possible presence of host galaxies associated with the quasars (Neugebauer $e t$ al. 1985 ) or because there were gaps in other observing programs.

The time intervals between the measurements varied from less than a month to more than $20 \mathrm{yr}$, but for all but two of the quasars, measurements from 1.25 to $2.2 \mu \mathrm{m}$ separated by at least $5 \mathrm{yr}$ were obtained. The distribution of the time intervals between all pairs of measurements at $2.2 \mu \mathrm{m}$ is shown in Fig. 5(a); as indicated, the distribution is dominated by observations of $3 \mathrm{C} 273$, which provide three quarters of the

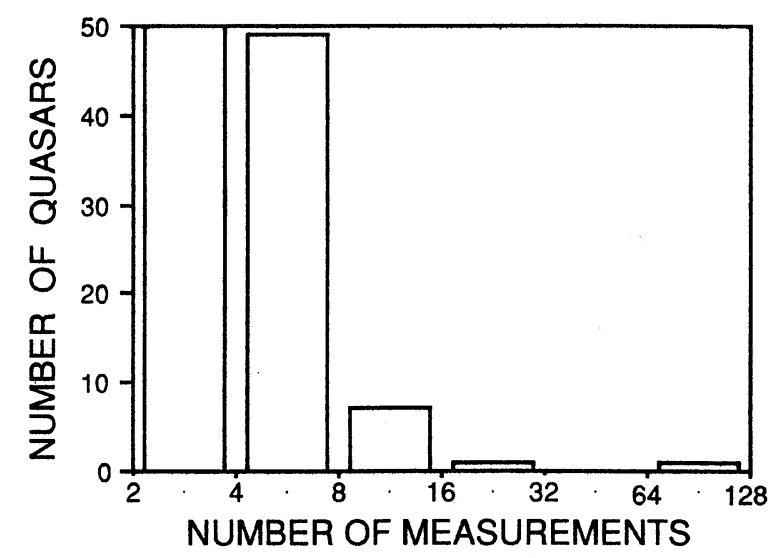

FIG. 4. The number of times each quasar in the sample has been observed at $2.2 \mu \mathrm{m}$ is indicated in the histogram. The vast majority have been observed two to four times, while one, $3 \mathrm{C} 273$, has been observed close to a hundred times.
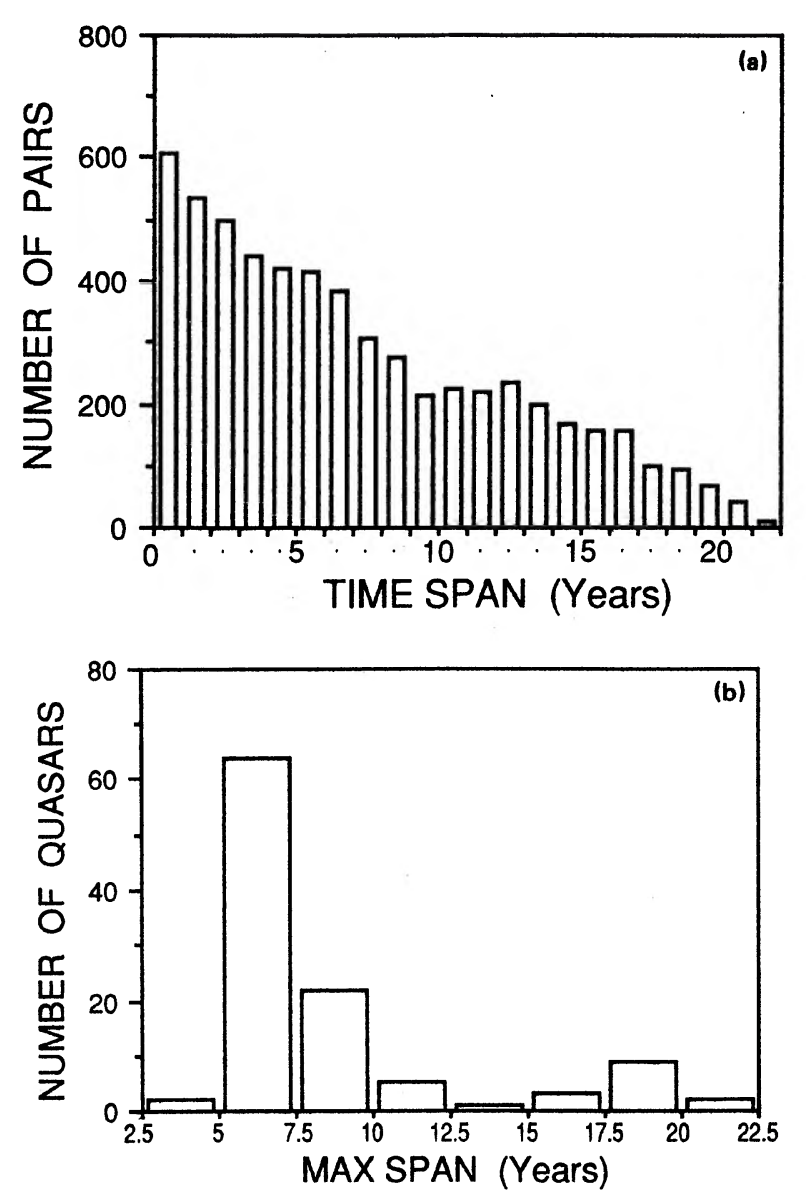

FIG. 5. (a) A histogram is presented of the distribution of time intervals between all pairs of observations at $2.2 \mu \mathrm{m}$. Of the 5800 total pairs of observations, 4465 have been obtained from observations of $3 \mathrm{C} 273$ on 95 nights and 406 have been obtained from observations of PG $0007+106$. (b) A histogram is given of the maximum time between observations of the quasars at $2.2 \mu \mathrm{m}$. The two quasars with the shortest coverage have been observed with time separations in excess of $4 \mathrm{yr}$.

pairs of observations. The distribution of the maximum time between measurements at $2.2 \mu \mathrm{m}$ for each of the 108 quasars is shown in Fig. 5(b).

\section{RESULTS-ANALYSIS}

Although it is well established that infrared emission plays an important role in the total luminosity of many quasars (see, e.g., Sanders et al. (1988) and references therein), the origin of the infrared emission, whether it is nonthermal synchrotron emission or thermal reradiation from dust grains heated by a central luminosity source, has been the subject of much debate. The bulk of the infrared emission in the quasars is emitted at wavelengths longer than $30 \mu \mathrm{m}$, so the near-infrared observations in this study do not sample the peak of the radiation. If, however, the emission in the near infrared is produced by a similar mechanism as that at the peak of the emission, large-amplitude rapid variations would favor a nonthermal interpretation, and it was hoped that the observations would provide an answer to the question of whether the infrared continuum emission in optically selected quasars is thermal or nonthermal. The thermal-re- 
laxation time is, however, short enough for expected grain sizes that thermal reradiation at $2.2 \mu \mathrm{m}$ would, since it measures hot grains near the luminosity source, follow a varying luminosity source with a timescale on the order of 1 or $2 \mathrm{yr}$. Thus the present observations at $2.2 \mu \mathrm{m}$ do not provide a sensitive discriminator between the models. At $10 \mu \mathrm{m}$, however, the shortest timescale for significant variations in the cooler dust cloud would be on the order of 100-200 yr, and the detection of significant variations on a timescale as short as $10 \mathrm{yr}$ would rule out that thermal emission from dust is a dominant contribution to the emission. These considerations will set the framework of the discussions of the different ways of analyzing the data presented below.

It is first illustrative to show the light curves for the six quasars in the sample-PG $0007+106,3 \mathrm{C} 273$, PG $1351+640$, PG $1535+547$, PG $1704+608=3$ CR 351, and PG $2302+029$-with $2.2 \mu \mathrm{m}$ measurements made on nine or more nights. From these light curves, which are given in Fig. 6, it is clear that the sampling represented in this study is mainly on the timescale of years. Short outbursts, such as detected in 3C 273 by Courvoisier et al. (1988), can easily go undetected in these, the most highly sampled of the present set. It is, however, seen that the six quasars represent quite different degrees of variability, although all show variability. In these six, as with the sample as a whole, the variations in the infrared are small with typical maximum variations on the order of $0.5 \mathrm{mag}$ at $2.2 \mu \mathrm{m}$. There is also some correlation between the variations at the different wavelengths. The light curves of 3C 273 have sufficient observations and time coverage that it is worthwhile to search for periodic features in the spectrum; this search and its implications are reported in Graham et al. (1988).

\section{PROBABILITY CALCULATIONS}

A presentation which takes into account the measurement uncertainty for each observation has been outlined by Penston and Cannon (1970). In this approach, the relative $\chi^{2}$ probability of variability $P_{\mathrm{v}}=1-P_{\mathrm{nv}}$ is calculated for each quasar. $\boldsymbol{P}_{\mathrm{nv}}$ is the probability that the hypothesis of nonvariability from the mean observed magnitude is correct. This was calculated from an evaluation of $\chi^{2}$ relative to the mean magnitude for the object and the uncertainties of the observations. In Table I and Fig. 7(a), the probability, $P_{\mathrm{v}}[2.2]$, of variability, relative to the mean of all the $2.2 \mu \mathrm{m}$ measurements obtained for that quasar, is given for each of the qua-
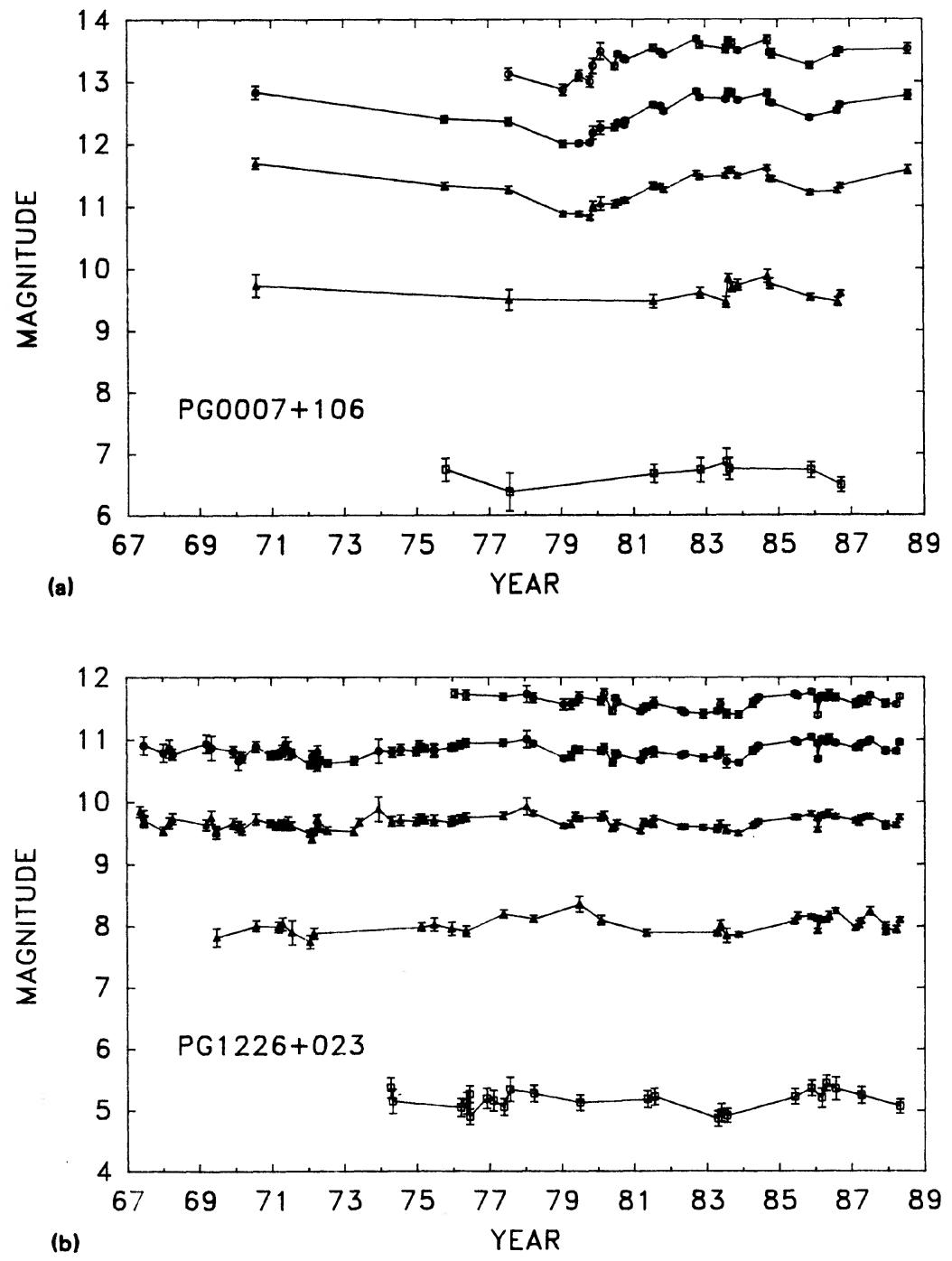

FIG. 6. (a) The time history of PG $0007+106$ is shown for the observations in the present study. The $1.25 \mu \mathrm{m}$ observations are indicated by light circles, the $1.65 \mu \mathrm{m}$ observations by dark circles, the $2.2 \mu \mathrm{m}$ by light triangles, the $3.7 \mu \mathrm{m}$ by dark triangles, and the $10.1 \mu \mathrm{m}$ observations by light squares. (b) The same as (a), but for PG $1226+023=3 \mathrm{C} 273$. (c) The same as (a), but for PG $1535+547$. (d) The same as (a), but for PG $1704+608$. (e) The same as (a), but for PG $2302+029$ (left) and for PG $1351+640$ (right). In order to help display the observations, 1 mag has been added to the observed $10.1 \mu \mathrm{m}$ magnitudes of PG $1351+640$. 

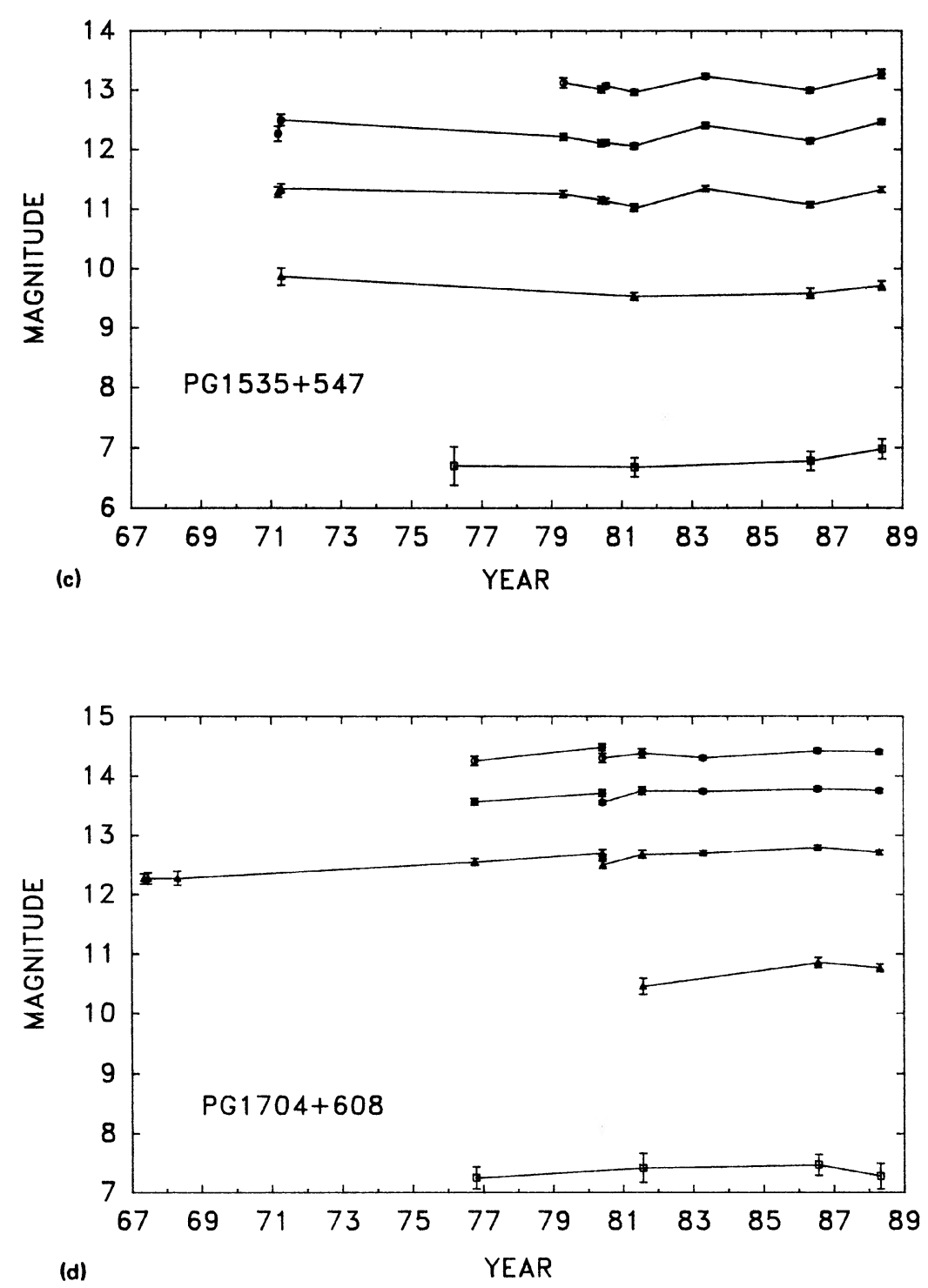

FIG. 6 (continued)
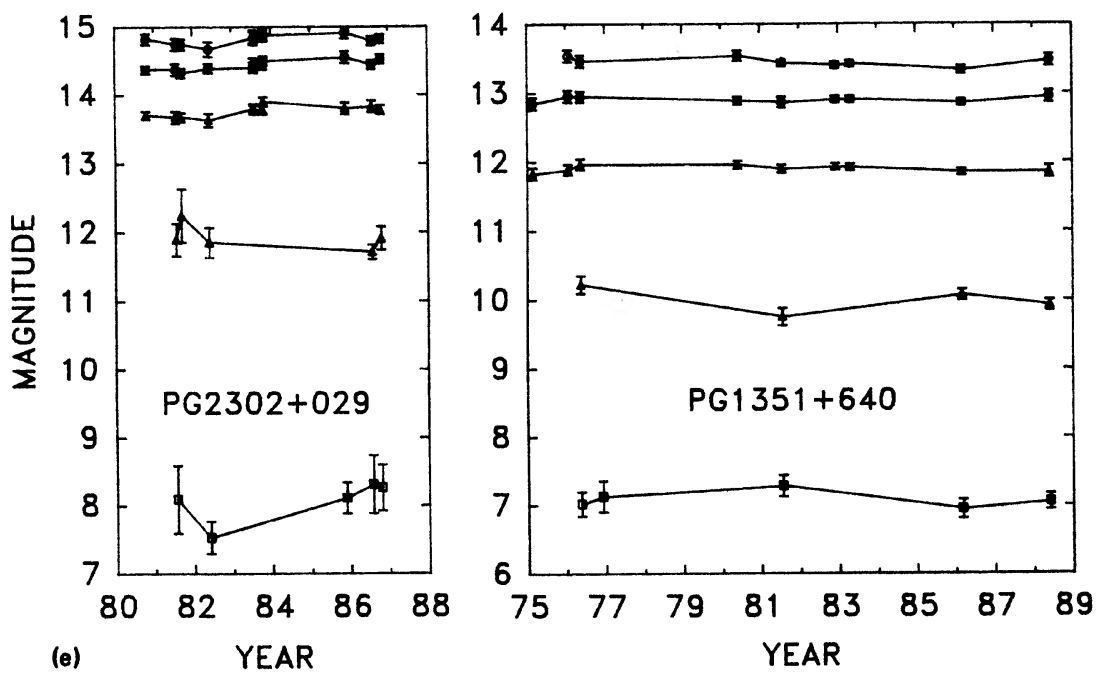

(C) American Astronomical Society - Provided by the NASA Astrophysics Data System 

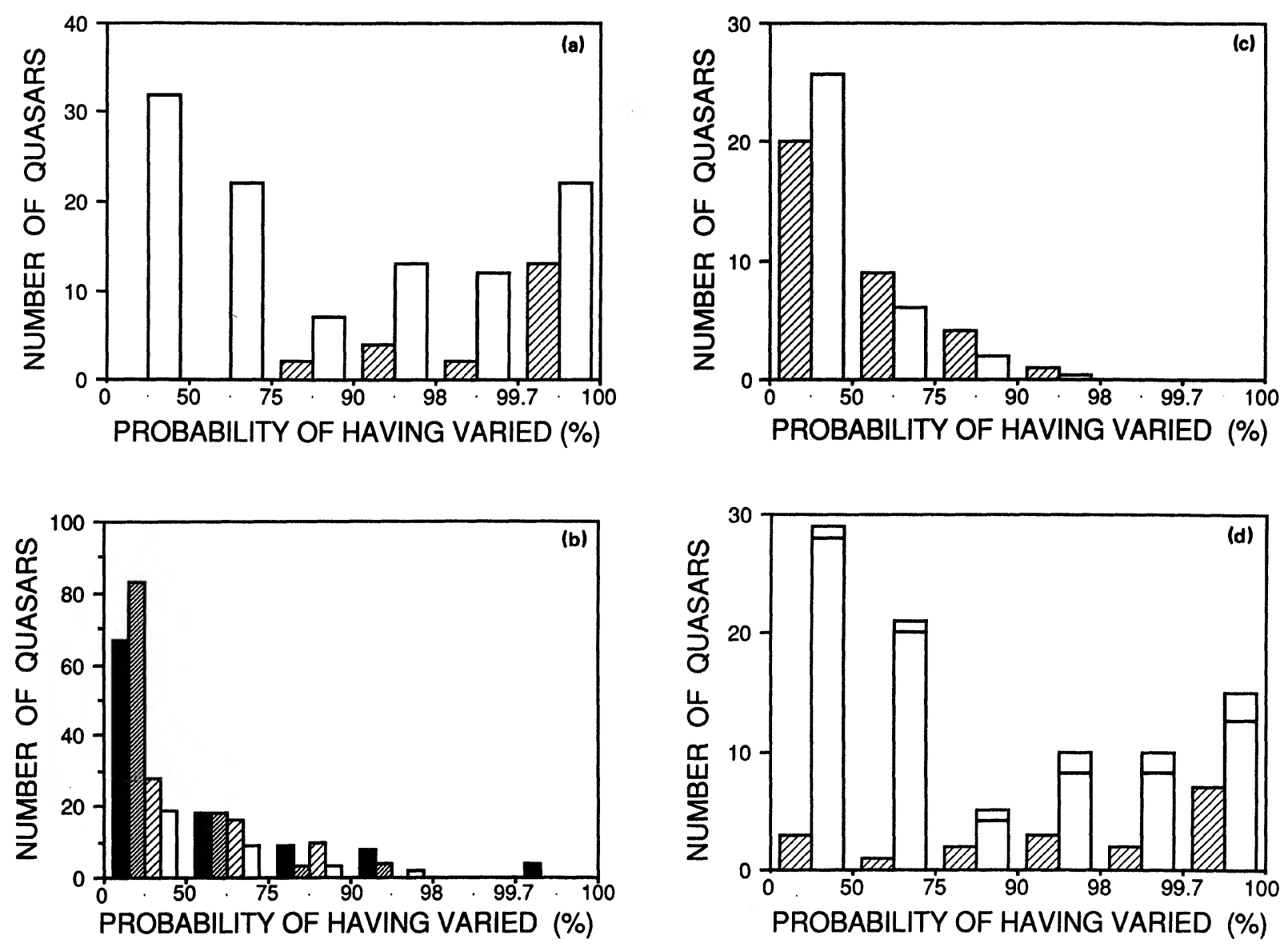

FIG. 7. (a) The probability of the PG quasars having varied at $2.2 \mu$ m during the observations reported here is shown in the distribution of the right-hand bars (light). The left-hand bars (hatched) give the distribution of the observations by Usher (1978) at $0.44 \mu \mathrm{m}$ of a sample of quasars in the Sandage Luyten field at $\alpha=8^{\mathrm{h}} 48^{\mathrm{m}}, \delta=+18^{\circ}$. The visual and near-infrared studies are comparable in time span and accuracy. (b) Histograms are given of the probability of having varied in the $J-K$ color (left bars, dark), in $H-K$ (second bars, narrow hatches), in $K-L$ (third bars, wide hatches) and in $K-N$ (right bars, light). Numerical simulations show that the distribution to the left of the figure is consistent with no true variation being present outside the uncertainties. (c) A histogram of the number of quasars that have a given probability of having varied at $10.1 \mu \mathrm{m}$ is given. The left-hand bars (hatched) correspond to the observations, while the right-hand bars (light) give the results of numerical simulations of constant $10 \mu \mathrm{m}$ magnitudes at the times of the observations and with variations based on the uncertainties in the observations. The latter numbers have been normalized so that the total number of quasars is the same. (d) The distribution of the probability of having varied at $2.2 \mu \mathrm{m}$ is divided according to the bolometric luminosity of the quasars. The bars on the left (hatched) represent the distribution of quasars with $\log [L(\mathrm{bol})]<12$, while the distribution of the right-hand bars (light) represents quasars with $\log [L($ bol $)]>12$. The ten quasars with reported flat spectrum radio spectra are above the lines in the right-hand bars; all these quasars have $\log [L(b o l)]>12$.

sars in the sample. These results are sensitive to the assumptions made of the size of the systematic uncertainties, but even when no systematic error was assumed in the measurement, the results are qualitatively the same as those seen in Fig. 7. The probability of having varied shows no significant correlation with the magnitude of the quasar.

Usher (1978) has applied a similar analysis procedure to 21 quasars in a field of faint blue objects in the SandageLuyten survey centered at right ascension $(1855)=8^{\mathrm{h}} 48^{\mathrm{m}}$, declination $(1855)=+18^{\circ}$. Although Usher used photographic surveys, the results of Usher's survey can in many ways be compared directly to those of the present survey, and a summary of Usher's results is included in Fig. 7(a). Usher analyzed the results from ten plates taken during a 6 yr span plus one additional plate which provided maxi- mum timescales of up to $23 \mathrm{yr}$, a maximum reached by only a few of the measurements of the present study. The uncertainties in the individual measurements quoted by Usher, basically $5 \%$ but sometimes as large as $50 \%$, are comparable to those in the present study at $2.2 \mu \mathrm{m}$. The quasars in Usher's study are all presumably radio "quiet."

Figure 7 (a) shows a striking difference between the two samples in that there are no quasars in Usher's (1978) sample with $P_{\mathrm{v}}[0.44]<0.8$, while half of the PG sample shows $P_{v}[2.2]<0.8$. This could be either a result of the increased time span observed by Usher, or it could reflect a true difference in the variability at optical and near-infrared wavelengths such as pointed out by Cutri et al. (1985). Usher (private communication) has indicated that the variability he observed was not dominated by the single plate providing 
the $23 \mathrm{yr}$ timescale. Furthermore, the analysis of the $2.2 \mu \mathrm{m}$ observations given below indicates that there is not a strong time dependence of the $2.2 \mu \mathrm{m}$ variability, and thus we favor the interpretation that, in fact, the variability in the infrared is often less than seen in the visible wavelengths. Both distributions share the property that the number of quasars definitely varying $\left(P_{\mathrm{v}}>99.5 \%\right)$ is relatively higher than the number with marginal variability; i.e., within the accuracy of these observations, the quasars in the sample either vary strongly or they do not.

The probabilities that each quasar varied in its near-infrared colors are presented in Fig. 7(b). There are few variations in color outside those attributable to the measurement uncertainties, although the sensitivity at 3.7 and $10.1 \mu \mathrm{m}$ is smaller than at $1.25-2.2 \mu \mathrm{m}$ because the uncertainties are larger. Numerical simulations of observations done at the times of the true observations show that the distribution on the left of Fig. 7(b) is completely consistent with no variations in the magnitudes outside random uncertainties with an amplitude similar to those observed. It is perhaps significant that the few cases of clear variability in the colors-for PG $0026+129$, PG $1001+054$, PG $1112+431$, and PG $1202+281=$ GQ Comae-occur for the shortest infrared wavelengths.

Because of the importance of the $10.1 \mu \mathrm{m}$ observations in distinguishing the mechanism for the infrared emission, the probabilities that the PG quasars varied at $10.1 \mu \mathrm{m}$ are displayed separately in Fig. 7(c) and included in Table I. In addition, the results of numerical simulations assuming that the variations are the result only of the measurement uncertainties are included in Fig. 7(c). Although the distribution of the observed probabilities is slightly broader than the simulations, the difference is not significant. The correlations of the 2.2 and $10.1 \mu \mathrm{m}$ observations of $3 \mathrm{C} 273$ shown below do, however, convincingly indicate that this quasar did vary at $10.1 \mu \mathrm{m}$ together with the $2.2 \mu \mathrm{m}$ variations.

In Fig. 7(d), the probability of variability is given for subsets of the sample with $L(\mathrm{bol})>10^{12} L_{\odot}$, the "quasars" (Schmidt and Green 1983), and those with $L(\mathrm{bol})<10^{12}$ $L_{\odot}$, the "low-luminosity" quasars. Two thirds of the low- luminosity quasars showed evidence for variability with a probability $P_{\mathrm{v}}[2.2]>90 \%$, while only one third of the luminous quasars exhibited similar variability.

The ten PG quasars that have published flat radio spectra are also identified in Fig. 7(d) and Table I. These ten sources were identified on the basis that their radio spectral index between 74 and $6 \mathrm{~cm}$ or 11 and $6 \mathrm{~cm}$ as given in Veron-Cetty and Veron (1988) or Dixon (1970) is greater than -0.4 . Seven of the ten quasars with flat radio indexes have a probability of having varied at $2.2 \mu \mathrm{m}$ greater than 0.9 , while only 40 of the remaining 98 quasars varied by a similar amount. Thus, although the differences in the measured variability of the quasars in the different subsets are not strong, there is evidence that the flat radio spectrum sources are more variable than the rest at infrared wavelengths.

As a further refinement, radio "quiet" quasars were separated from radio "loud" quasars on the somewhat arbitrary basis, derived from an examination of the PG sample by Sanders et al. (1988), of the ratio of the flux densities at $6 \mathrm{~cm}$ wavelength to those at $1.25 \mu \mathrm{m}$ being less than 3160 or exceeding 31600 . This definition, which resulted in 22 radio loud cases and 14 "ambiguous" cases, will be adopted throughout the rest of this paper. Radio observations have not been published for eight quasars in the sample, and those quasars without published radio fluxes are lumped together with the radio "quiet" quasars. The radio observations used here are largely those of Kellerman et al. (1988) or are taken from the compilation by Veron-Cetty and Veron (1987). It should be noted that this division is necessarily incomplete; as pointed out by Antonucci and Barvainis (1988), radio "quiet" quasars have both steep and flat radio spectra. Of the 72 radio "quiet" quasars in the PG sample, 30 have a probability exceeding $90 \%$ of having varied during these observations. The connection between the radio spectrum and the probability of having varied in the infrared is thus independent of the detailed definition of the radio properties.

It is possible that the relatively short time spans of the observations contributes to the lack of observed variability. Figure 8 shows the sample averages of the probabilities for variation at $2.2 \mu \mathrm{m}, P_{\mathrm{v}}(2.2)$, as a function of the time inter-

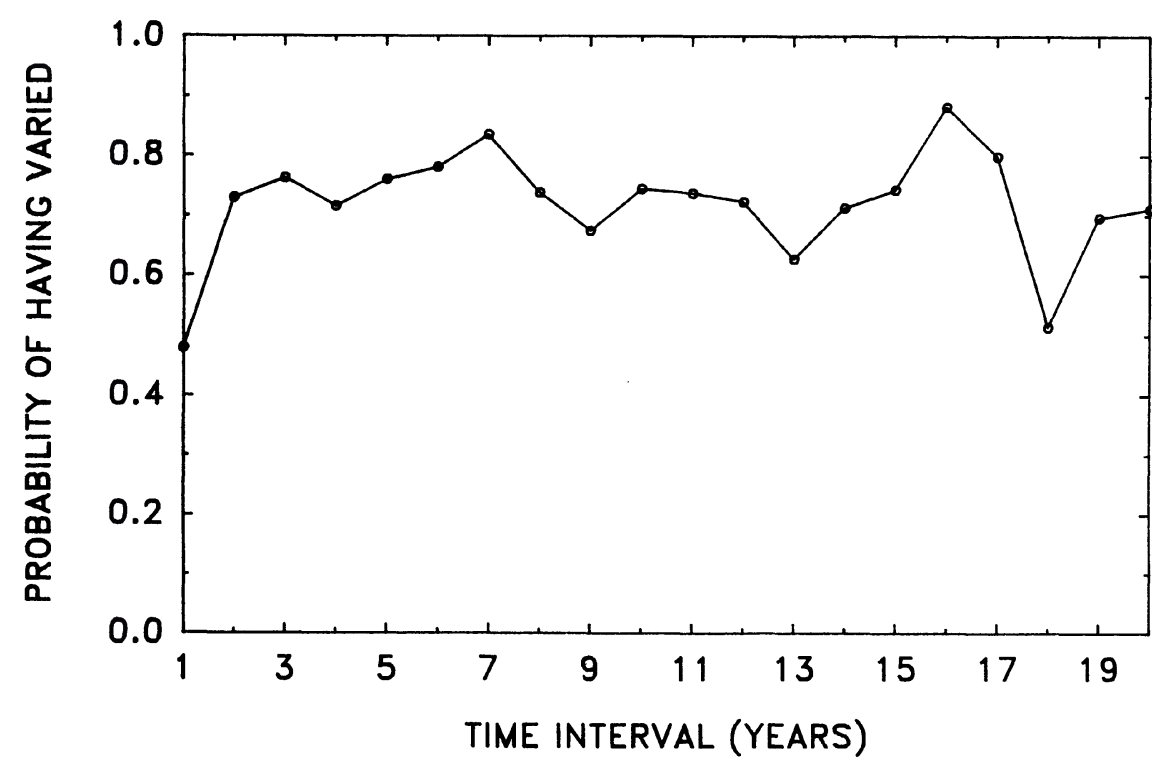

FIG. 8. The average probability for a pair of measurements made with a given time interval between them is given for those quasars which have a probability of having varied during the survey exceeding 0.8 . The dispersions in the probabilities is large, ranging from $<0.5$ to 1 . 
val between measurements for those quasars which showed a probability greater than 0.8 of having varied at $2.2 \mu \mathrm{m}$. For each pair of measurements for each of the variable quasars in the sample, the probability of variability was calculated as described above. All the probabilities for each quasar were then averaged with a weighting such that each quasar had equal total weight over all the time intervals. The dispersion in the individual contributions to the average is large. For most time intervals, there were cases where, even with this sample of identified variable quasars, the probability of having varied was less than $50 \%$. The relative constancy of the averages beyond about $1 \mathrm{yr}$ indicates that the variations appear at all timescales sampled.

All observations since 1967 are included in Fig. 7. A subset of the observations which is potentially less biased by selection effects is one restricted to observations made after 1980 when the infrared and visual measurements of Neugebauer et al. (1987) were started. The sample of more recent observations is also potentially less susceptible to long-term drifts in the photometric system. A comparison of the post1980 observations and the whole set of observations is given in Fig. 9. From this figure it is clear that the more restricted sample, which only spans times up to $8 \mathrm{yr}$, has qualitatively the same variability pattern as does the larger sample. This finding is consistent with the results of Fig. 8.

In order to sharpen some of the trends apparently present in Fig. 7, the post-1980 observational results are displayed in a different format in Fig. 10. The observations included in this figure have also been chosen, by selecting the variability at appropriate observed wavelengths as a function of the quasar redshift, so that observations at approximate rest frame wavelengths of $1.25 \mu \mathrm{m}$ (Fig. 10 (a)) and $1.65 \mu \mathrm{m}$ (Fig. 10 (b)) are represented. Although the scatter dominates the figures, systematic trends remain between the radio-type/ spectral index, the bolometric luminosity, and the probability that the quasar has varied; these trends are illustrated in Table II, which shows the average bolometric luminosity $L$ (bol) for different subsets of the data in Fig. 10.

Perhaps the most striking feature of both Fig. 10 and Table II is the fact that the flat spectrum sources, of which three quarters exhibit a high probability of having varied, have significantly higher average luminosities than do the quasars

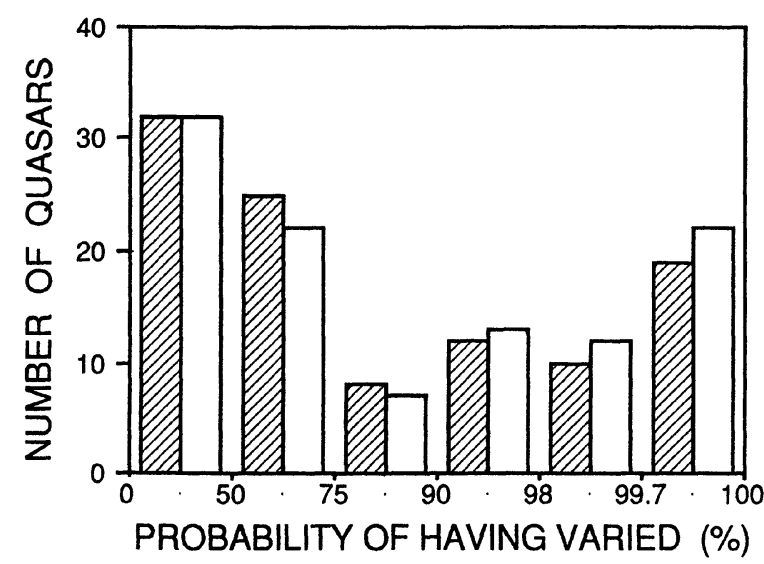

FiG. 9. The distribution of the probability of having varied at $2.2 \mu \mathrm{m}$ is shown for all measurements in this study (right-hand bars, light) and for measurements made after 1980 (left-hand bars, hatched). The latter are presumably less subject to selection effects.
TABLE II. Systematic trends.

\begin{tabular}{|c|c|c|c|c|}
\hline & \multicolumn{4}{|c|}{$\log \left[L(\mathrm{bol}) / L_{\odot}\right]$} \\
\hline & \multicolumn{2}{|c|}{$1.25 \mu \mathrm{m}$} & \multicolumn{2}{|c|}{$1.65 \mu \mathrm{m}$} \\
\hline & Quiet/Steep & Flat & Quiet/Steep & Flat \\
\hline $\begin{array}{l}P_{\mathrm{v}}>0.9 \\
P_{\mathrm{v}}<0.1\end{array}$ & $\begin{array}{l}12.2 \pm 0.1 \\
12.8 \pm 0.1\end{array}$ & $\begin{array}{c}12.8 \pm 0.2 \\
-\end{array}$ & $\begin{array}{l}12.1 \pm 0.1 \\
12.8 \pm 0.2\end{array}$ & $\begin{array}{c}12.7 \pm 0.23 \\
-\end{array}$ \\
\hline
\end{tabular}

that are radio "quiet" or have steep radio spectral indexes. This difference is also obvious from the histogram of Fig. 7 (d) and is presently the first observable difference between flat radio spectrum and steep radio spectrum sources in the near infrared.

In addition, if the ten quasars with flat radio spectral indexes are excluded, the average bolometric luminosity of those quasars that have definitely varied in this $8 \mathrm{yr}$ period is significantly less than that for those which have not varied. Thus there is a tendency, admittedly of low statistical significance, for the lower-luminosity quasars to have varied more than the higher-luminosity quasars. This tendency is in agreement with, but does not require or prove, the interpretation that the infrared emission is thermal reradiation from dust. In this case, the grains radiating at a given wavelength are closer to the source if they are heated by a lower-luminosity source and thus are more likely to vary on a short timescale; the timescale for variability should increase as (luminosity $)^{(1 / 2)}$. An alternative interpretation may be that sources with smaller bolometric luminosities have less dust than higher luminosity quasars and that consequently the short-wavelength variations are not as severely damped out due to the cooler grains at larger distances from the central source.

\section{WAVELENGTH CORRELATIONS}

Although Fig. 7(b) shows that the probability of color changes in the PG quasars having occurred is generally low, another, perhaps less quantitative, presentation indicates that there are possibly some trends in the variability with wavelength. In Figs. 11(a)-11(j), the magnitude differences between all pairs of measurements for each object in the sample at $1.25,1.6,3.7$, and $10.1 \mu \mathrm{m}$ are plotted against the difference of the $2.2 \mu \mathrm{m}$ magnitudes measured on the same pair of nights. The sense of the figures is such that points in the lower left corner of the figure represent brightening in both wavelengths. For this presentation, the measurements were restricted to those with uncertainties in the individual magnitudes of less than $15 \%$. In addition, all measurements at 3.7 and $10.1 \mu \mathrm{m}$ with uncertainties less than $0.5 \mathrm{mJy}(3.7 \mu \mathrm{m})$ and $7.5 \mathrm{mJy}(10.1 \mu \mathrm{m})$ are included, making these, in effect, flux-limited samples. All the individual pairs of measurements meeting these criteria are included in the plots, but the observations of PG $0007+106$ and 3C 273 are plotted separately because the number of observations of these quasars is so much larger than the number of observations of the other quasars. It was not possible to find a meaningful correlation at $10.1 \mu \mathrm{m}$ other than for $3 \mathrm{C} 273$.

Figure 11 confirms that the average changes in the 1.25 and $1.65 \mu \mathrm{m}$ magnitudes of the PG quasars are approximately the same as those at $2.2 \mu \mathrm{m}$. The slopes of the individual datasets are given in Table III; observations with absolute 


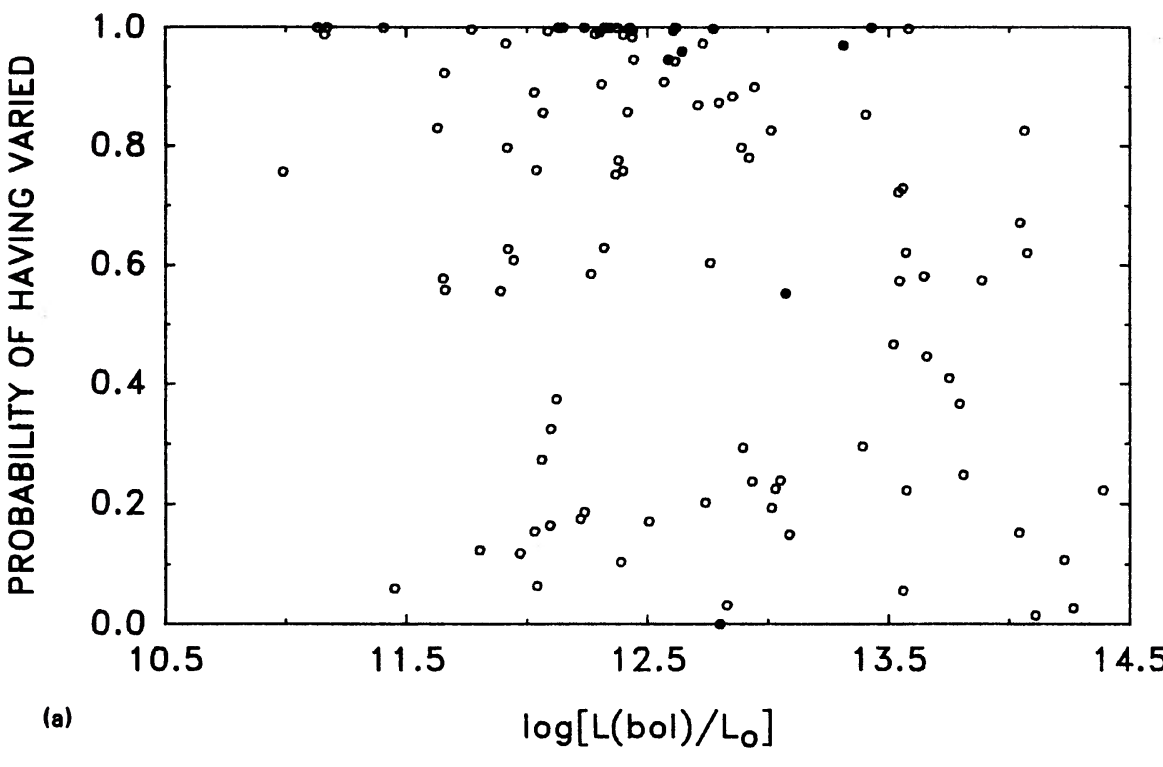

FIG. 10. (a) The probability of having varied at a rest wavelength near 1.25 $\mu \mathrm{m}$ is plotted against the bolometric luminosity. The quasars with flat radio spectra are indicated by dark circles, while the rest are represented by light circles. (b) Same as Fig. 10(a), but for a rest wavelength near $1.65 \mu \mathrm{m}$.

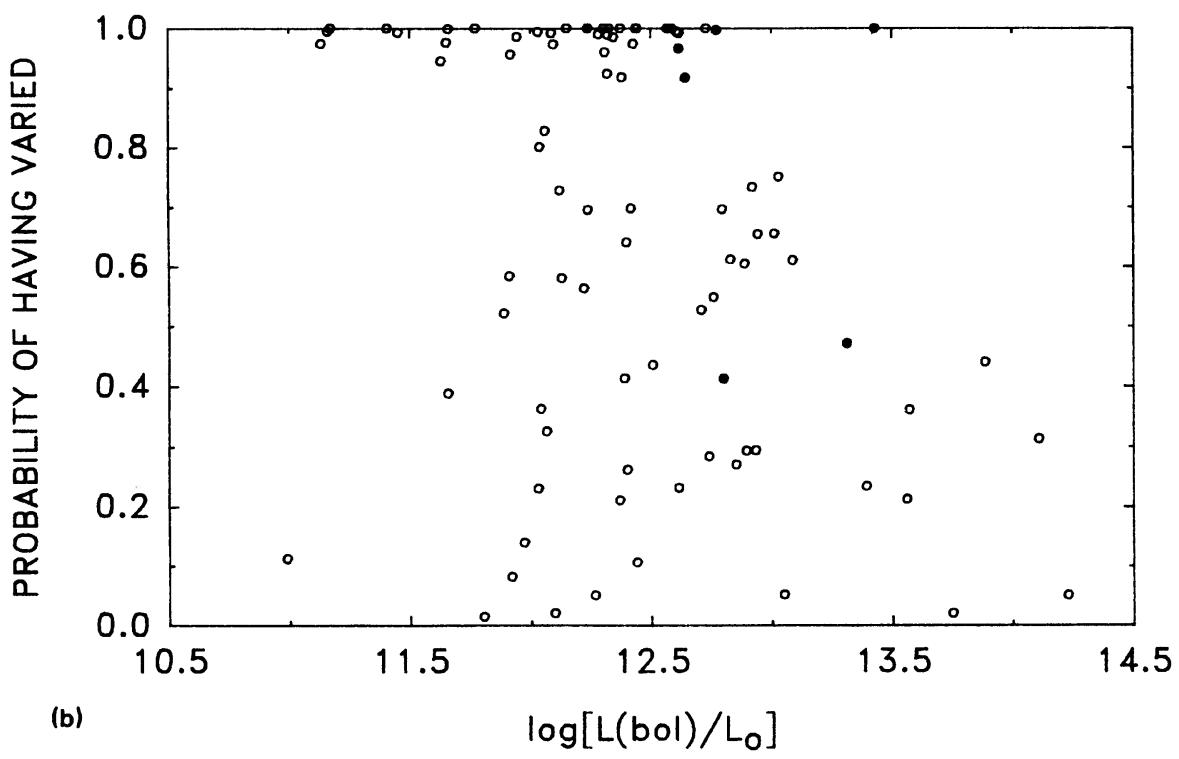

changes in the $2.2 \mu \mathrm{m}$ magnitudes of less than 0.08 mag were not included in the calculation of the slopes. The correlation coefficients for the fits vary between 0.75 and 0.97 .

An examination of Table III shows that the two quasars with sufficient measurements to define individual color correlations differ in their color changes from each other and from the mean of the entire sample, indicating true differences among the quasars in this sample. Although PG $0007+106$ shows significantly the largest changes in slope among the three sets of data, 3C 273 is definitely different from the mean of the rest, another indication that it cannot be considered as a template for all quasars. All three datasets show an increase in slope with wavelength and for 3C 273 the $10.1 \mu \mathrm{m}$ variations are clearly greater than those at $2.2 \mu \mathrm{m}$. For this quasar, at least, the $3.7 \mu \mathrm{m}$ measurements indicate that the relative changes at this wavelength are also greater than those at $2.2 \mu \mathrm{m}$, again indicating that the long-wavelength components of the continuum of this quasar have greater variations than do the components producing the shorter-infrared-wavelength emission.

Although there seem to be systematic trends in the amplitudes for variation, the correlations evident in Fig. 11 are certainly sufficiently good to indicate that the emission is a common response to the same stimulus, such as would be the case if the near-infrared emission results from the hottest grains from within a distribution of grains at various distances from the luminosity source and at various temperatures. The increased variations at the longer wavelengths which are evident in 3C 273 are, however, difficult to reconcile with the simplest thermal model for the infrared emission and probably rule out thermal emission from dust grains dominating the continuum in that quasar. If the variations come about purely from a short-wavelength source heating the grains, this result implies that some other component must be present in the emission at near-infrared wavelengths. It should be noted that a similar conclusion 

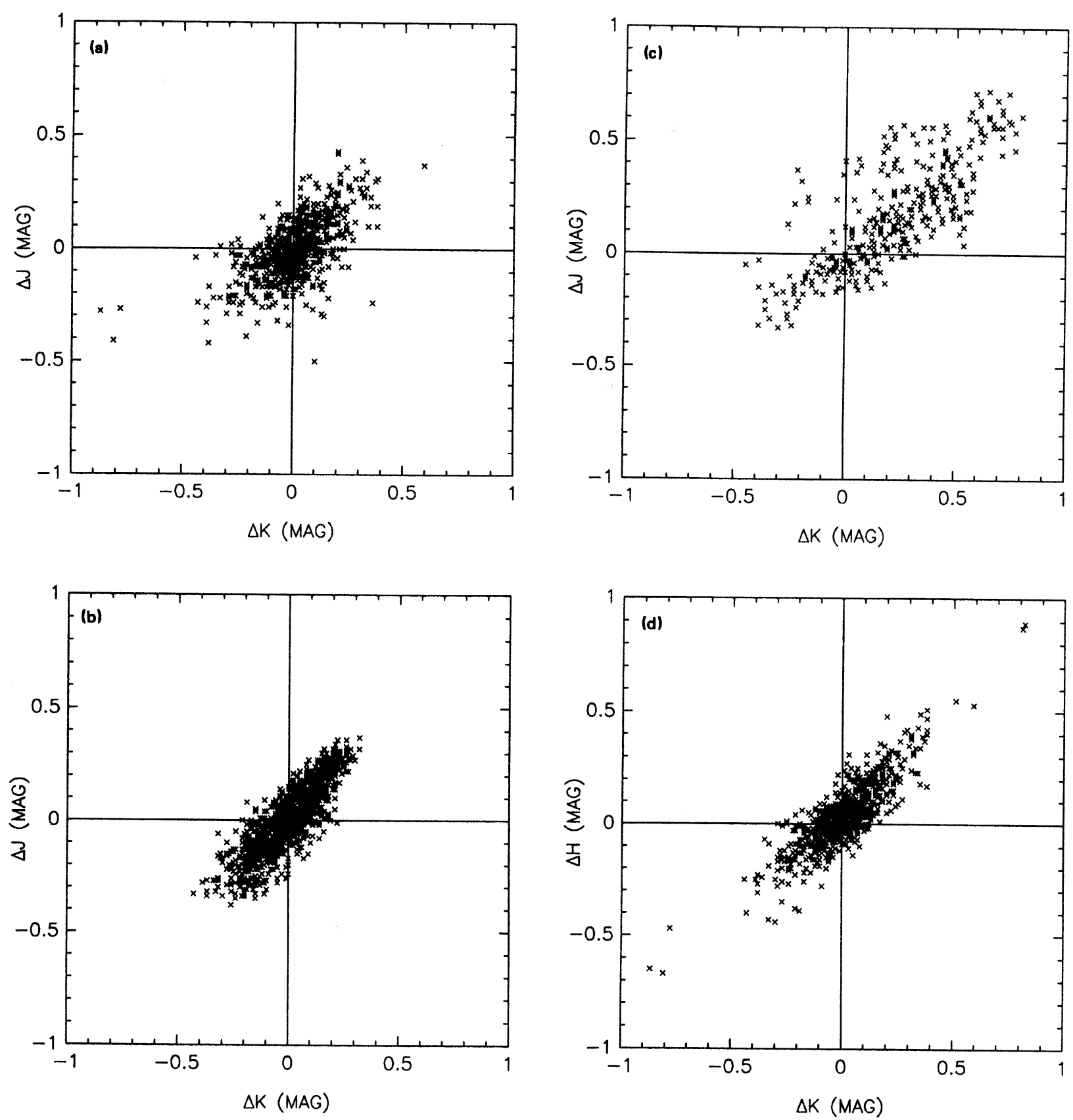

FIG. 11. (a) The change, $\Delta(J)$, in the $1.25 \mu \mathrm{m}$ magnitudes of the PG quasars is shown as a function of the change, $\Delta(K)$, in the $2.2 \mu \mathrm{m}$ magnitudes. All pairs of measurements with uncertainties $0.15 \mathrm{mag}$ or less are included. Measurements of all the PG quasars are included except those for 3C 273 or PG $0007+106$. (b) Same as (a) except the measurements are only of 3C 273. (c) Same as (a) except the measurements are only for PG $0007+106$. (d) Same as (a) except measurement of the change $\Delta(H)$ at $1.65 \mu \mathrm{m}$ is shown. (e) Same as (b) except measurement of the change $\Delta(H)$ at $1.65 \mu \mathrm{m}$ is shown. (f) Same as (c) except measurement of the change $\Delta(H)$ at $1.65 \mu \mathrm{m}$ is shown. (g) Same as (a) except measurement of the change $\Delta(L)$ at $3.7 \mu \mathrm{m}$ is shown. (h) Same as (b) except measurement of the change $\Delta(L)$ at $3.7 \mu \mathrm{m}$ is shown. (i) Same as (c) except measurement of the change $\Delta(L)$ at $3.7 \mu \mathrm{m}$ is shown. (j) Same as (b) except measurement of the change $\Delta(N)$ at $10.1 \mu \mathrm{m}$ is shown. 

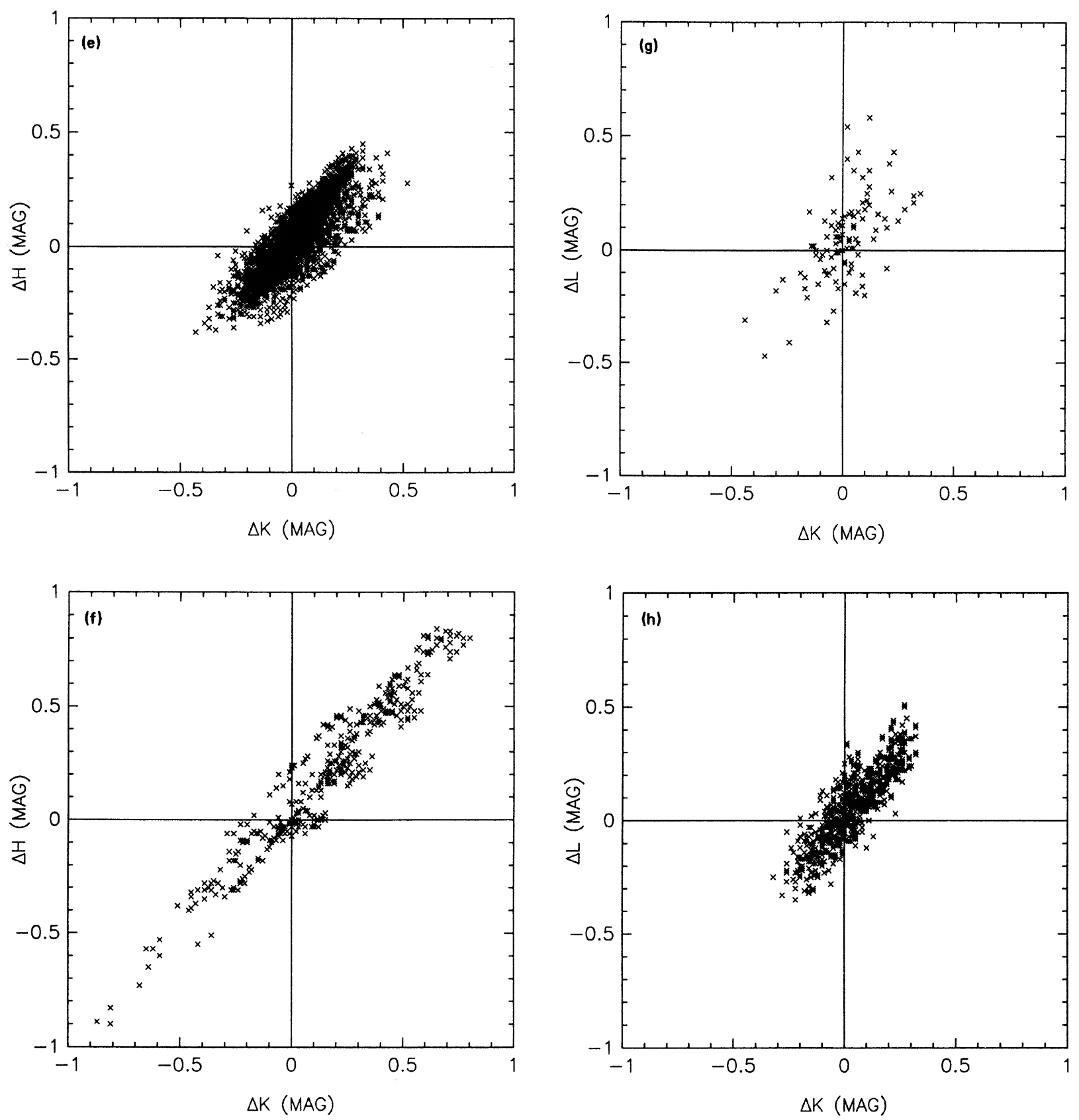

FIG. 11 (continued) 

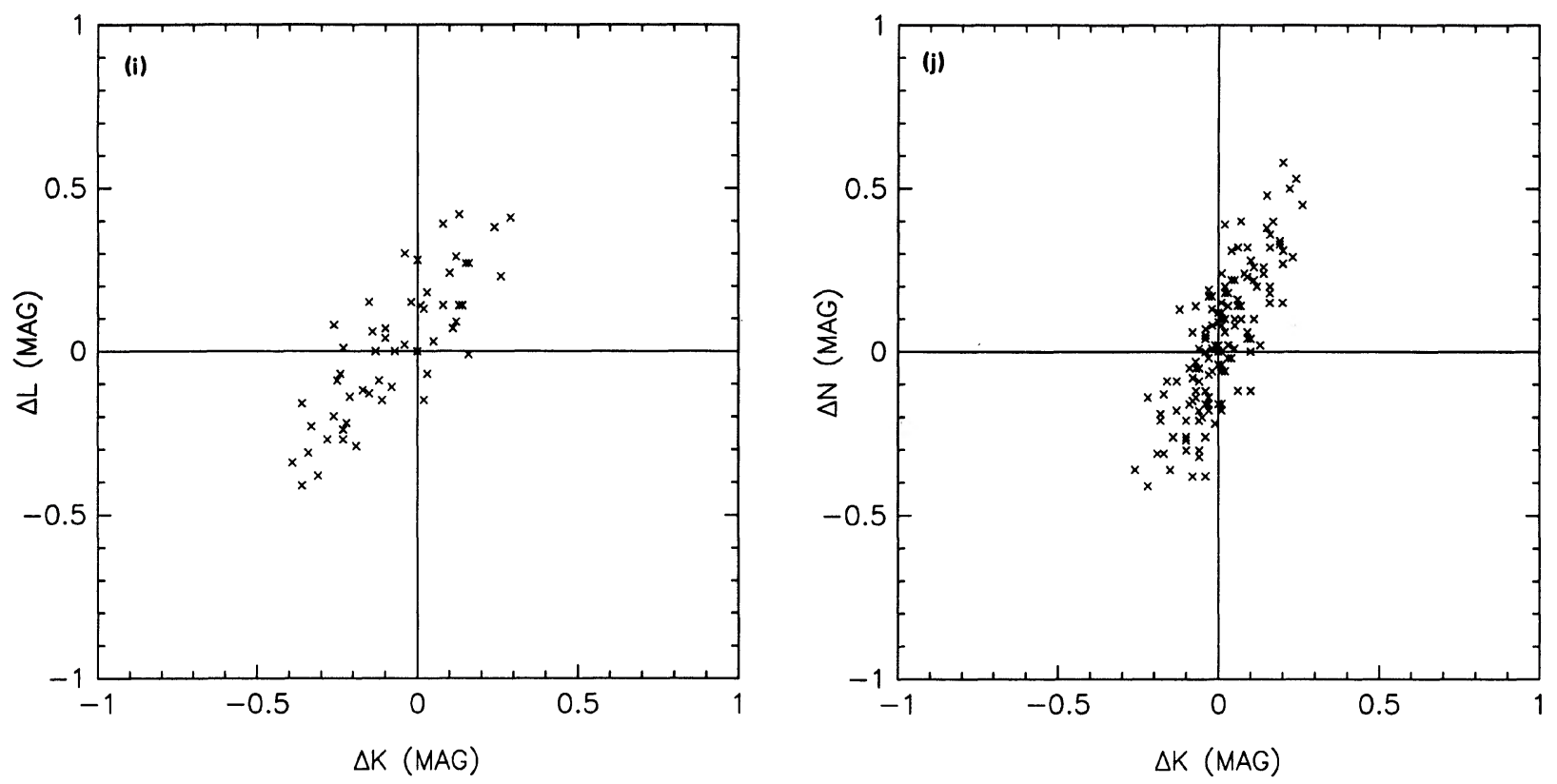

FIG. 11 (continued)

was drawn by Rieke and Lebofsky (1979) based on the outburst in the $10 \mu \mathrm{m}$ flux density in early 1972 .

The observations were again divided into those associated with either radio loud or radio quiet quasars. No strong correlation between the distributions in Fig. 11 and this radio property was evident.

\section{DISPERSION/LUMINOSITY CORRELATION}

In Fig. 12, the dispersions for the $2.2 \mu \mathrm{m}$ measurements for each PG quasar are plotted against the luminosity of that quasar at $2.2 \mu \mathrm{m}$. Only the $2.2 \mu \mathrm{m}$ observations with less than $15 \%$ uncertainties have been included in Fig. 12. For reference, the dispersion calculated for the 107 observations at $2.2 \mu \mathrm{m}$ of the star Bertaud b (Bertaud et al. 1973) over approximately $20 \mathrm{yr}$ was $0.04 \mathrm{mag}$.

The presentation of Fig. 12 is patterned after that of Angione (1973), who studied the historical light curves over about $50 \mathrm{yr}$ of 20 quasars at optical wavelengths; his sample

TABLE III. Slopes of individual datasets.

\begin{tabular}{lccc}
\hline \hline & \multicolumn{3}{c}{ Average Color Changes } \\
\cline { 2 - 4 } & $3 C 273$ & PG $0007+106$ & rest \\
\hline$\Delta J / \Delta K$ & $0.97 \pm 0.02$ & $0.68 \pm 0.03$ & $0.72 \pm 0.04$ \\
$\Delta H / \Delta K$ & $0.95 \pm 0.01$ & $1.03 \pm 0.02$ & $0.87 \pm 0.02$ \\
$\Delta L ' / \Delta K$ & $1.05 \pm 0.02$ & $1.01 \pm 0.09$ & $0.80 \pm 0.12$ \\
$\Delta N / \Delta K$ & $1.56 \pm 0.11$ & - & - \\
\hline \hline
\end{tabular}

includes a mixture of radio "quiet" and both steep and flat radio spectrum radio loud quasars. His observations are included in Fig. 12, which shows that the PG quasars have very roughly the same range of variability in the near infrared as the most heterogeneous sample exhibits at visible wavelengths. The denser concentration of low standard deviations in the infrared data may represent a true limit on the range of variability of many of the PG quasars, but reflects as well the increased accuracy of the infrared detectors above the photographic monitoring. In Fig. 12, two quasars -the well-known optically violent variables $3 \mathrm{C} 345$ and 3C 454.3-stand out from the rest of the optical sample with dispersions larger than $0.5 \mathrm{mag}$. Similarly, two of the PG quasars-PG $0049+171=$ Mrk 1148 and PG $1001+054$ -show dispersions $>0.4 \mathrm{mag}$, which is almost sufficient to place them in the class of violent variables. Both are radio quiet rather than flat spectrum radio loud sources. As a comparison, PG $0007+106$ and 3C 273 have dispersions at 2.2 $\mu \mathrm{m}$ of 0.24 and $0.09 \mathrm{mag}$.

Fahlman (1977) has interpreted the observations at optical wavelengths in terms of a shot noise model of the variability. He has also pointed out that, even after the optically violent variables are discounted, the observations of Angione (1973) fall into two separate groups of differing activity, the lower typified by $3 \mathrm{C} 48$ and the more active group by 3C 273. This separation is strengthened by considering, as done in Fig. 13, the dispersion in the luminosities of the quasars as a function of the fractional dispersion in the luminosi- 


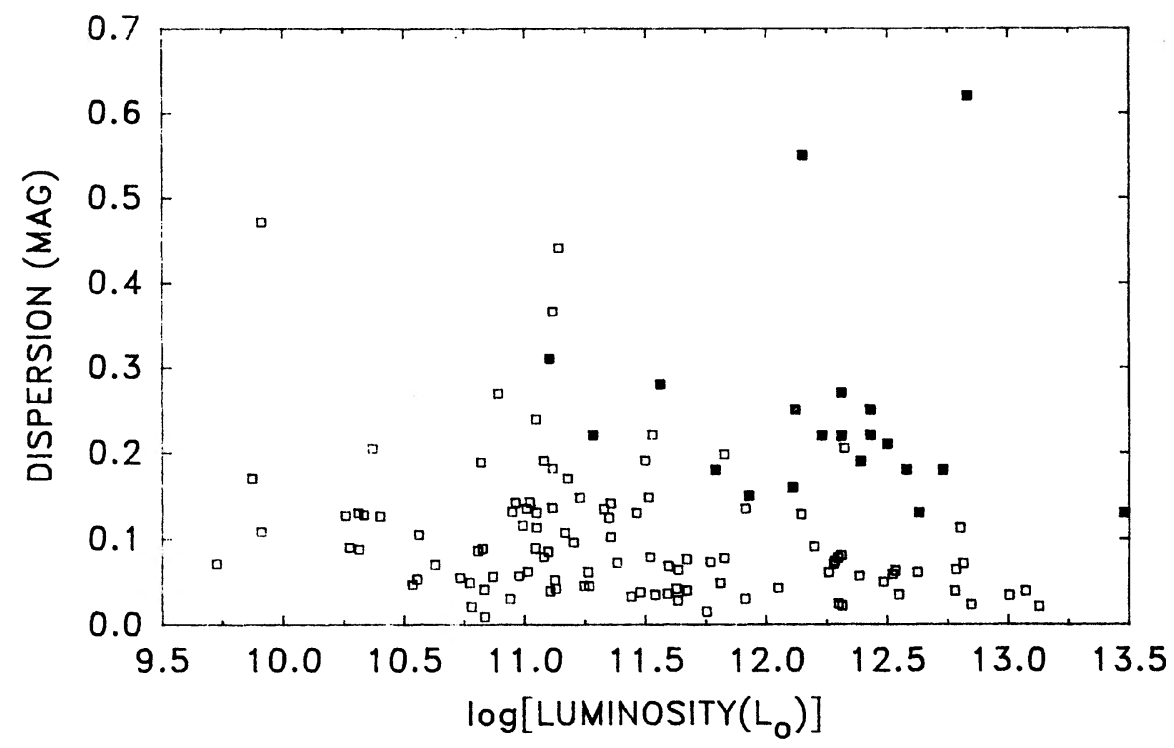

FiG. 12. The dispersions for the $2.2 \mu \mathrm{m}$ measurements for each PG quasar are plotted against the luminosity of that quasar at $2.2 \mu \mathrm{m}$ using light squares. Only observations with less than $15 \%$ uncertainties have been included. The dispersion calculated for 107 observations at $2.2 \mu \mathrm{m}$ of the star Bertaud b (Bertaud et al. 1973) over 19 yr was 0.04 mag. The dark squares correspond to the dispersions derived from the historical light curves over about $50 \mathrm{yr}$ of a different set of 20 quasars at optical wavelengths (Angione 1973).

ty. If the shot noise picture of Fahlman applies, all quasars with the same amplitude of outbursts will lie along a line parallel to that drawn in Fig. 13. The infrared observations of the PG sample show no distinct grouping, but rather show a continuum of values even when plotted as in Fig. 13. Thus, if a shot model applies to the source driving the infrared emission, the amplitudes of the shots apparently form a continuous set rather than two distinct bunches.

\section{TIME DEPENDENCE}

There were not enough observations to establish significant time histories of all the quasars in the sample, and in fact, most of the quasars have been observed only three or four times in this study (see Fig. 4). As a result, the time dependence of those few quasars with sufficient observations to give meaningful behaviors are first described, after which an attempt is made to represent the time behavior of the entire sample.

\section{STRUCTURE FUNCTION OF INDIVIDUAL QUASARS}

The timescale of possible variations of individual quasars with a sufficient number of observations can be studied through the use of the structure function SF $(d t)$ (see, e.g., Simonetti, Cordes, and Heeschen 1985). For a time interval between observations of $d t, \mathrm{SF}(d t)$ is defined as

$$
\mathrm{SF}(d t)=\left\langle[\operatorname{mag}(t)-\operatorname{mag}(t+d t)]^{2}\right\rangle .
$$

The angular brackets denote an ensemble average, and mag $(t)$ is the magnitude at any wavelength measured at time $t$. This function, which is similar to the auto- and crosscorrelation functions, roughly characterizes the intensity of variations on timescales less than or comparable to $d t$. The structure functions at those wavelengths where observations on 10 or more nights were obtained are shown in Fig. 14. Since the samples were obtained at unequal intervals, the data were binned in 2 or $4 \mathrm{yr}$ and the average structure function in that bin calculated. The uncertainties shown are in

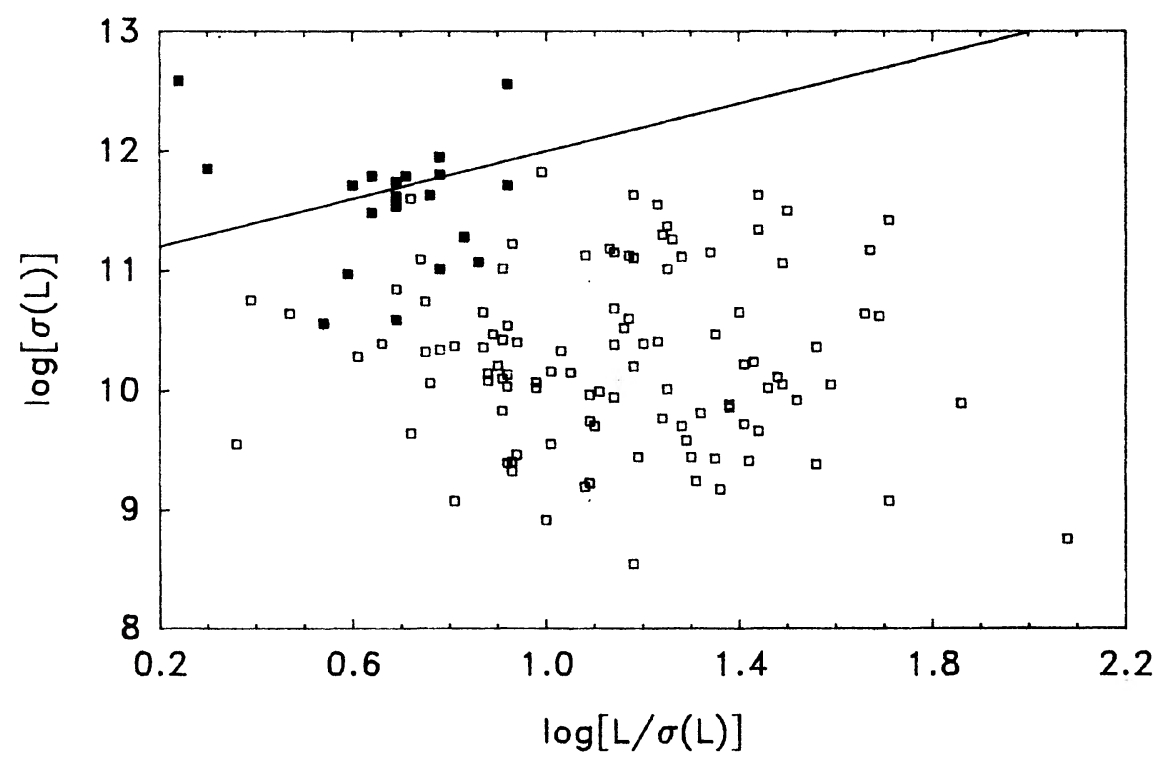

Fig. 13. The dispersions at $2.2 \mu \mathrm{m}$ for the PG quasars (light squares) and the dispersions observed by Angione (1973) (dark squares) for a set of 20 quasars are displayed in the format of Fahlman (1977). The line corresponds to a fixed shot amplitude in the model of Fahlman; the amplitude has been adjusted to pass through the dispersions of a group of quasars of relatively high optical activity typified by $3 \mathrm{C} 273$. 

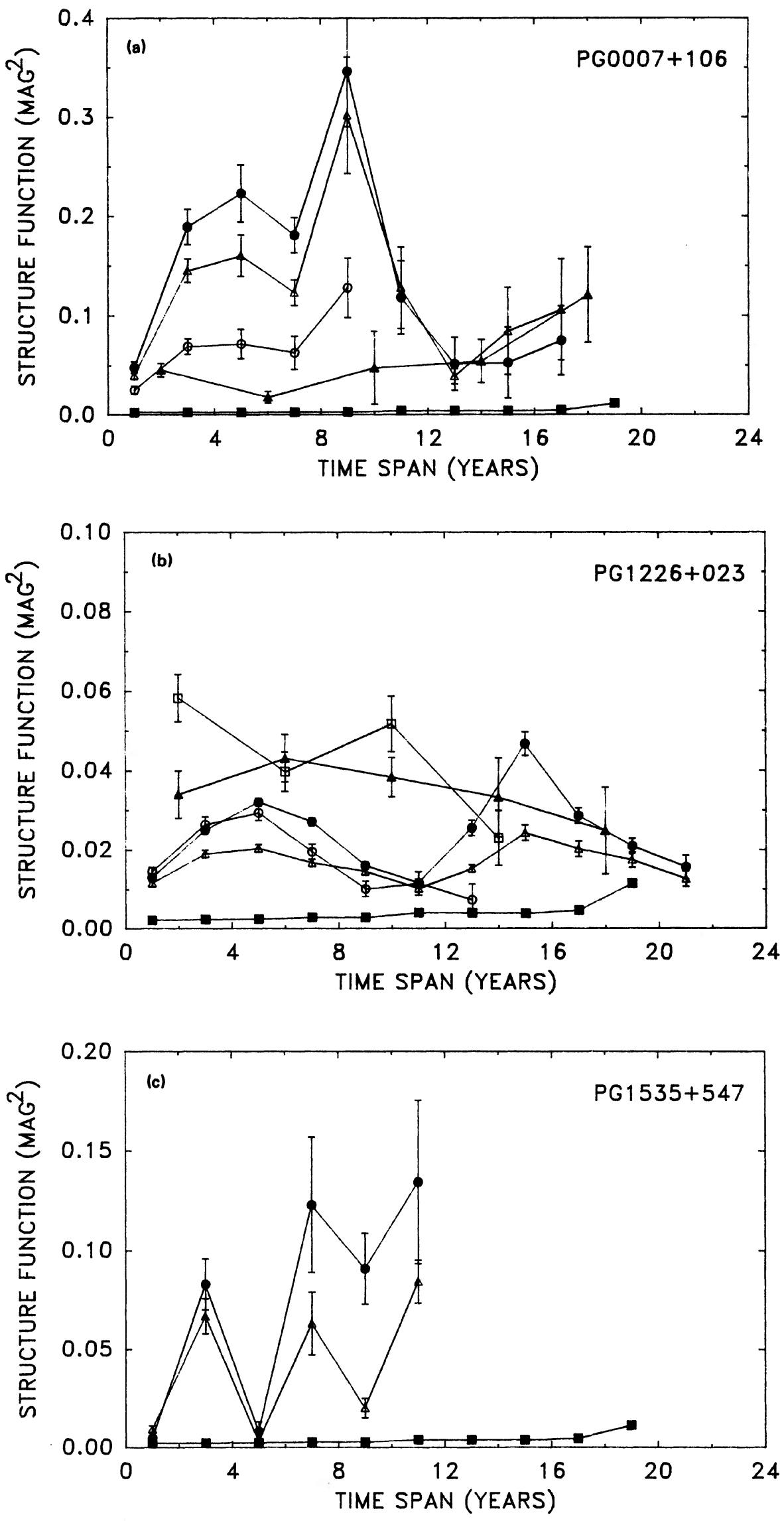

FIG. 14. (a) The structure functions for the quasar PG $0007+106$ at various wavelengths are given. The light circles represent $1.25 \mu$ m data, dark circles 1.65 $\mu$ m data, light triangles $2.2 \mu \mathrm{m}$ data, and dark triangles $3.7 \mu \mathrm{m}$ data. The dark squares at the bottom represent the structure function for $2.2 \mu \mathrm{m}$ observations of the star Bertaud $b$ (Bertaud $e t$ al. 1973). (b) Same as (a) except for the quasar $3 \mathrm{C} 273$. The structure function for $10.1 \mu \mathrm{m}$ is indicated by light squares. Note the change in the vertical scale. (c) Same as (a) except for the quasar PG $1535+547$. (d) Same as (a), except for the quasar PG $1704+608$. (e) Same as (a) except for the quasar PG $2302+029$. 


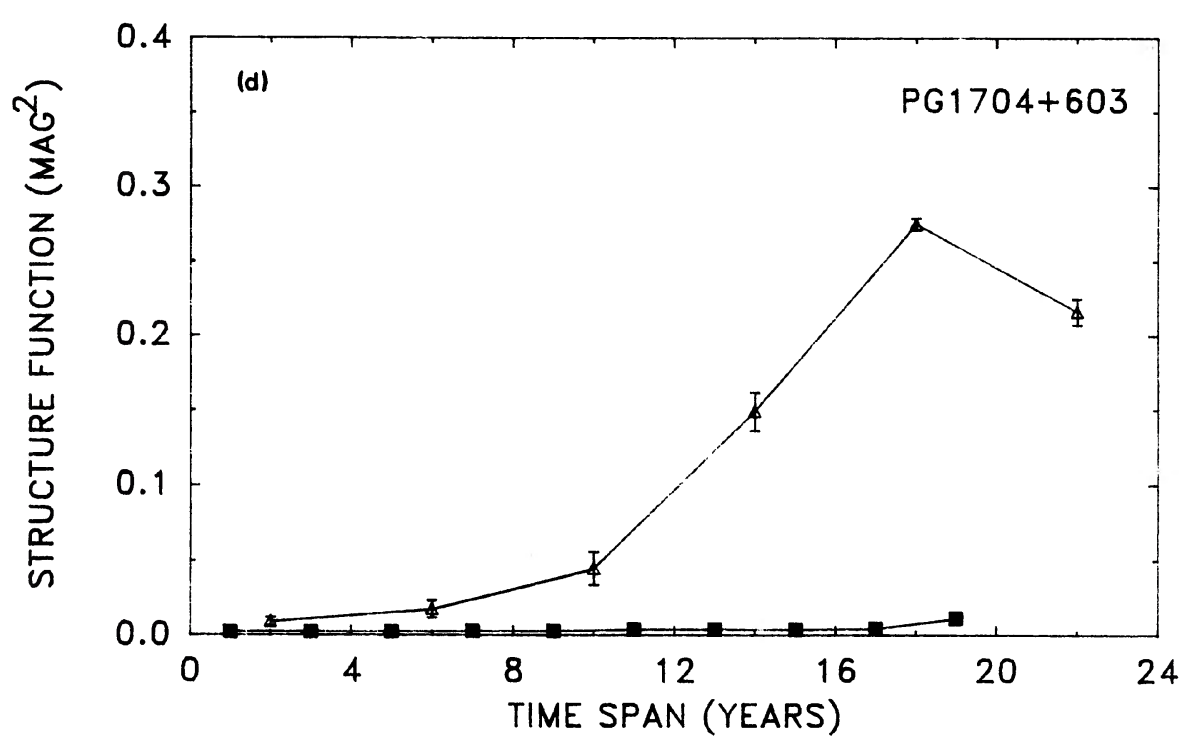

FIG. 14 (continued)

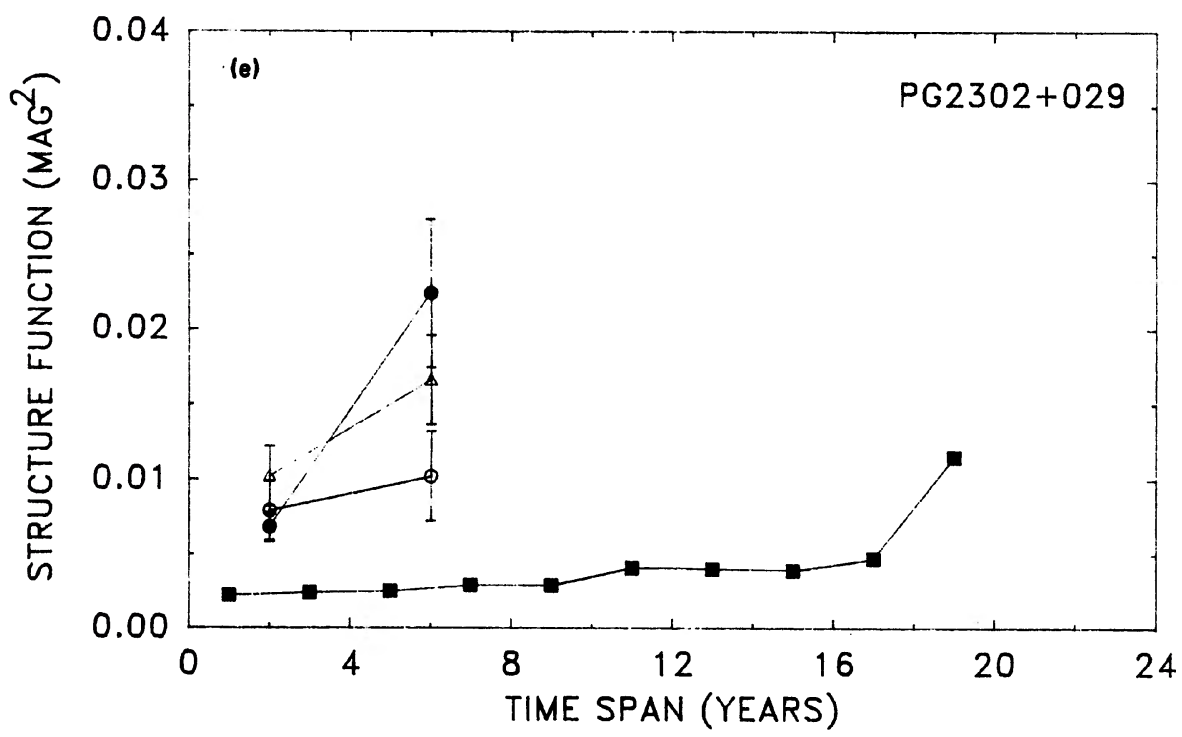

the mean structure function and are calculated from the dispersion of the individual determinations of $\operatorname{SF}(d t)$. The structure function for the 107 observations of the star Bertaud b (Bertaud et al. 1973) at $2.2 \mu \mathrm{m}$ is included in each plate of Fig. 14. It is immediately obvious that the values of the structure function of Bertaud $b$ lie well below those observed for the quasars and that thus there is real variability in the quasars. It is also obvious from Fig. 14 that the different quasars have a variety of time behavior.

The interpretation of the structure function is confused by the paucity of observations and the very uneven sampling. In order to interpret Fig. 14, datasets were therefore constructed with simulated observations at the dates of the $2.2 \mu \mathrm{m}$ observations of the quasars in Fig. 14. Random noise with an amplitude dependent on the actual uncertainties of the quasar was added to an assumed input light profile, and at least five runs of each assumed input profile and random noise were carried out. A constant input resulted in structure functions of about 0.004 , closely resembling that of Bertaud b. A single step of 0.2 mag corresponding to an extreme systemat- ic change in the photometry in 1973 also resulted in a structure function of mean amplitude close to 0.04 , well below the peak amplitudes observed in Fig. 14. A peak such as seen in PG $0007+106$ could be produced by an outburst of amplitude about $1.5 \mathrm{mag}$, which occurred around 1980 and decayed with a time constant of slightly less than a year. The rise in PG $1704+608$ is consistent with an outburst in the early 1970's, which decayed with a time constant in excess of 50 yr. More complicated structure, such as seen in 3C 273, can be reproduced by a series of discrete outbursts separated by about $5 \mathrm{yr}$. Although it is clear that none of these possible input patterns is unique, it is well established that significant variability on timescales of years is present.

\section{AVERAGE STRUCTURE FUNCTIONS}

As a heuristic approach to dealing with the large number of objects with only a few observations per object, an average structure function was calculated from the observations of all the quasars. This analysis gives a valid description of the 
sample if all the quasars in the sample behave in the same way, but it is important to remember that any trends obtained can come about because of anomalous variations of individual quasars and do not necessarily reflect the behavior of the entire sample.

At each wavelength, each pair of observations for each quasar was used to evaluate Eq. (1), and the average for all the quasars with a lag time within either a 2 or 4 yr time bin was determined. The results are shown in Fig. 15. In order to avoid the averages being dominated by the few quasars with many observations, the individual contributions were weighted in the average so that each quasar has equal weight in the function as a whole. In addition, the average structure function was also calculated by weighting the average function for each quasar contributing to a bin equally. Both types of weighting methods give qualitatively the same results. The uncertainties shown are the uncertainties in the averages calculated from the dispersion in the individual contributions for a particular time interval. Observations at 1.25$3.7 \mu \mathrm{m}$ were limited to those with uncertainties less than $15 \%$; at $10.1 \mu \mathrm{m}$ all observations with uncertainties less than $20 \%$ were included.

The most striking feature of Fig. 15 is the sharp rise at timescales on the order of $10 \mathrm{yr}$, which is consistent with an outburst occurring once during the time of measurement of the quasars. This result, however, is largely due to the observations of PG $1001+054$ where measurements at 1.25 , 1.65 , and $2.2 \mu \mathrm{m}$ in 1976 were brighter by approximately 1 mag than subsequent measurements made after 1981. If this one quasar is not included in the dataset, the average structure function for the bin centered on $10 \mathrm{yr}$ goes from $0.18 \pm 0.04 \mathrm{mag}^{2}$ to $0.03 \pm 0.01 \mathrm{mag}^{2}$.

For lag times shorter than about $8 \mathrm{yr}$, the average structure functions at $1.25,1.65$, and $2.2 \mu \mathrm{m}$ are essentially the same, in agreement with the presentation of Fig. 11. The structure functions for the longer-wavelength observations again indicate the presence of variations, but are less informative because of the shorter base lines and higher noise levels.

\section{SUMMARY}

The observations of a sample of optically selected quasars show that there is significant variability in the near infrared with an amplitude of several tenths of a magnitude. A significant fraction of the quasars have not, however, varied at $\mathbf{2 . 2}$ $\mu \mathrm{m}$ by more than the $5 \%$ measurement uncertainty over a 5 $10 \mathrm{yr}$ period, in marked contrast to the behavior of quasars in the visible. The dispersions in the near infrared fill in the total range from zero to several tenths of a magnitude. There are at least two examples of quasars, both of which are radio "quiet" quasars, which show large amplitude variability at $2.2 \mu \mathrm{m}$.

The histories of those few quasars that have been measured sufficiently often to provide a time sequence show that there is a range of behaviors in the variability. The structure functions of these quasars can be explained by one or more outbursts of approximately $1 \mathrm{mag}$ in amplitude, which decay with time constants from one to several years. The average structure function of all the quasars shows a small rise, which is typical of timescales of 5-10 yr. The uniformity of the mean probability with time is probably indicative of a series of short outbursts driving the variations. The mean luminosity of those quasars that gave evidence for variability is less than that for those that showed little evidence of variability.

The colors of the quasars have remained essentially the same as the quasars varied. In more detail, variations at 2.2 $\mu \mathrm{m}$ were accompanied by approximately the same, but slightly smaller, variations at $1.25,1.65$, and $3.7 \mu \mathrm{m}$ for the bulk of the sample. The quasar 3C 273 showed larger variations at $3.7 \mu \mathrm{m}$ and at $10.1 \mu \mathrm{m}$ relative to its variations at 2.2 $\mu \mathrm{m}$ than did the rest of the quasars in this sample.

Seven out of ten flat spectrum radio loud quasars in the sample had more than a $90 \%$ probability of varying at 2.2 $\mu \mathrm{m}$. In contrast, $40 \%$ of the remaining quasars show more than $90 \%$ probability of having varied.

The observations strengthen the clear implication that the mechanism predominant in the near infrared is different from that responsible for the emission at visible wavelengths or that the variations in the infrared represent severely damped reactions to outbursts in the optical. The observations, however, cannot be used to prove or disprove the hypothesis that the infrared emission from quasars is thermal emission. Some observations, such as the color correlations and the dependence of the variability on luminosity, are consistent with the near-infrared emission consisting of radi-

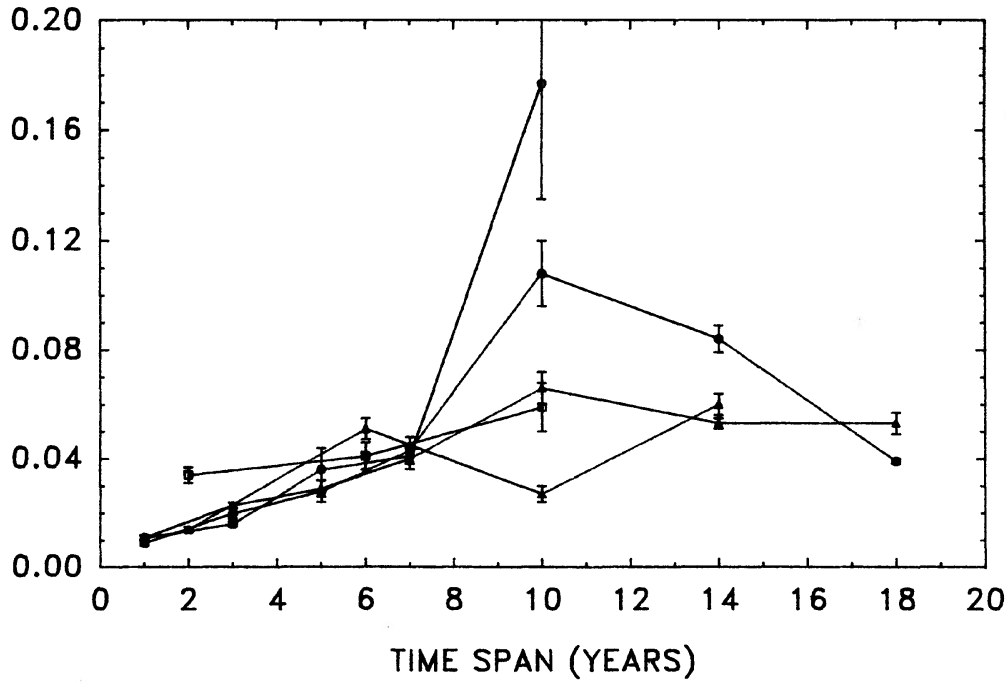

FIG. 15. An heuristic average structure function for the measurements of the sample as a whole is shown. Each quasar has been given equal weight in the averages. The bins are either 2 or $4 \mathrm{yr}$ in length; the midpoint of each bin is plotted. The wavelength designation is the same as in Fig. 14. The light circles represent $1.25 \mu \mathrm{m}$ data, dark circles $1.65 \mu \mathrm{m}$, light triangles $2.2 \mu \mathrm{m}$ data, dark triangles $3.7 \mu \mathrm{m}$ data and light squares $10.1 \mu \mathrm{m}$. At 1.65 and $2.2 \mu \mathrm{m}$ all time intervals greater than $16 \mathrm{yr}$ are included in the last bin at $18 \mathrm{yr}$. 
ation from hot grains being heated by a time-varying luminosity source. Other observations, in particular the presence of increased variability at long wavelengths, are harder to explain with a simple model invoking purely thermal emission in the near infrared.

Variations in the $10.1 \mu \mathrm{m}$ observations of 3C 273 appear to have been definitely detected. The straightforward statistical analysis, summarized in Table I, shows an $80 \%$ probability of variability for 3C 273 . In addition, the strong correlation between the $10.1 \mu \mathrm{m}$ variation and $2.2 \mu \mathrm{m}$ variation, as shown in Fig. 11(j), convincingly shows that these fluxes vary together. Because of these $10.1 \mu \mathrm{m}$ variations, we conclude that the $10.1 \mu \mathrm{m}$ continuum from this quasar cannot be due to thermal emission from heated dust.

The $10.1 \mu \mathrm{m}$ observations of the other PG quasars cannot convincingly be shown to demonstrate variability. Figure 7 (c) shows that there is not a substantial excess of sources having a high probability of having varied at $10.1 \mu \mathrm{m}$ beyond that expected from measurement errors. Thus the observations presented here are consistent with, but do not prove or even suggest, a model of thermal emission by dust contributing significantly to the $10.1 \mu \mathrm{m}$ emission of the other quasars.

These observations are consistent with the interpretation of the energy distributions of the PG quasars discussed by Sanders et al. (1988), who find that the radio-quiet quasars show an infrared excess, and attribute this excess to thermal emission by dust. The sources with flat spectra, such as $3 \mathrm{C}$ 273 , show no such excess, and can be explained as synchrotron radiation extending from the radio through the infrared.

A project of this length cannot have been done without the help of many co-workers through the years and we thank them for their assistance, but especially E. Becklin. The night assistants at the 200 in. Telescope, J. Carrasco, S. Staples, and G. Tuton, deserve much credit for their help and patience. We thank them and the entire mountain staff of Palomar Observatory. The maintenance of the large database was the responsibility of J. Bennett. We acknowledge useful conversations with S. Phinney, J. Graham, D. Sanders, and P. Usher. J. Bregman and J. Simonetti kindly introduced us to the concept of the structure function. We thank George Rieke for pointing out that in 1972 he had detected an outburst in 3C 273 at $10 \mu \mathrm{m}$ coincident with an increase in $2.2 \mu \mathrm{m}$. Financial support over the years has been provided by grants from NASA, the NSF, and the California Institute of Technology.
Angioni, R. J. (1971). Astron. J. 76, 25.

Angioni, R. J. (1973). Astron. J. 78, 353.

Antonucci, R., and Barvainis, R. (1988). Astrophys. J. Lett. 332, L13.

Bertaud, C., Wlerick, G., Veron, P., Dumortier, B., Bigay, J., Paturel, G., Duruy, M., and deSaevsky, P. (1973). Astron. Astrophys. 24, 357.

Bregman, J. N., et al. (1986). Astrophys. J. 301, 708.

Courvoisier, T. J.-L., Turner, M. J. L., Robson, E. I., Gear, W. K., Staubert, R., Blecha, A., Bouchet, P., Falomo, R., Valtonen, M., and Terasranta, H. (1987). Astron. Astrophys. 176, 197.

Courvoisier, T. J.-L., Robson, E. I., Hughes, D. H., Blecha, A., Bouchet, P., Krisciunas, K., and Schwarz, H. (1988). Preprint.

Cutri, R. M., Wisniewski, W. Z., Rieke, G. H., and Lebofsky, M. J. (1985). Astrophys. J. 296, 423.

Dixon, R. S. (1970). Astrophys. J. Suppl. 20, 1.

Elias, J. H., Frogel, J. A., Matthews, K., and Neugebauer, G. (1982). Astron. J. 87, 1029.

Fahlman, G. G. (1977). Astrophys. J. 211, 649.

Graham, J., Neugebauer, G., Phinney, S., and Matthews, K. (1988). In preparation.

Green, R. F., Schmidt, M., and Liebert, J. (1986). Astrophys. J. Suppl. 61, 305.

Kellerman, K. I., Sramek, R., Shaffer, D. B., Schmidt, M., and Green, R. F. (1988). In preparation.

Lebofsky, M. J., and Rieke, G. H. (1980). Nature 284, 410.

Lu, P. K. (1972). Astron. J. 77, 829.

McGimsey, B. Q., Smith, A. G., Scott, R. L., Leacock, R. J., Edwards, P. L., Hackney, R. L., and Hackney, K. R. (1975). Astron. J. 80, 895.

Neugebauer, G., Matthews, K., Soifer, B. T., and Elias, J. H. (1985). As-

\section{REFERENCES}

trophys. J. 298, 275.

Neugebauer, G., Oke, J. B., Becklin, E. E., and Matthews, K. (1979). Astrophys. J. 230, 79.

Neugebauer, G., Green, R. F., Matthews, K., Schmidt, M., Soifer, B. T., and Bennett, J. (1987). Astrophys. J. Suppl. 63, 615.

Peach, J. V. (1969). Nature 222, 439.

Penston, M. V., and Cannon, R. D. (1970). R. Obs. Bull. No. 159.

Rieke, G. H., and Lebofsky, M. J. (1979). Annu. Rev. Astron. Astrophys. $17,477$.

Robson, E. I., Gear, W. K., Brown, L. M. J., Courvoisier, T. J.-L., Smith, M. G., Griffin, M. J., and Blecha, A. (1986). Nature 323, 134.

Robson, E. I., Gear, W. K., Clegg, P. E., Ade, P. A. R., Smith, M. G., Griffin, M. J., Holt, I. G., Radostitz, J. V., and Howard, R. J. (1983). Nature 305, 194.

Sanders, D., Neugebauer, G., Phinney, S. E., Soifer, B. T., Green, R. F., and Matthews, K. (1988). In preparation.

Schmidt, M., and Green, R. F. (1983). Astrophys. J. 269, 352.

Scott, R. L., Leacock, R. J., McGimsey, B. Q., Smith, A. G., Edwards, P. L., Hackney, K. R., and Hackney, R. L. (1976). Astron. J. 81, 7.

Selmes, R. A., Tritton, K. P., and Wordsworth, R. W. (1975). Mon. Not. R. Astron. Soc. 170, 15.

Simonetti, J. H., Cordes, J. M., and Heeschen, D. S. (1985). Astrophys. J. 296, 46.

Smith, P. S., Balonek, T. J., Elston, R., and Heckert, P. A. (1987). Astrophys. J. Suppl. 64, 459.

Tokunaga, A. (1984). Astron. J. 89, 172.

Usher, P. D. (1978). Astrophys. J. 222, 40.

Veron-Cetty, M. P. and Veron, P. (1987). ESO Sci. Rep. No. 5. 